\title{
SYNTHESIS AND PHYSICOCHEMICAL PROPERTIES OF NEW ESTOLIDE ESTERS AS POTENTIAL BIOLUBRICANT BASE OIL
}

\author{
SENG SOI HOONG*; MOHD ZAN ARNIZA*; NEK MAT DIN NIK SITI MARIAM*; ABU HASSAN NOOR \\ ARMYLISAS*; SOOK WAH TANG*; TUAN NOOR MAZNEE TUAN ISMAIL* and SHOOT KIAN YEONG*
}

\begin{abstract}
In this study, plant oil-derived oleic acid was modified chemically to generate estolide esters that displayed low pour points and good oxidation stability. Specifically, oleic acid was reacted with hydrogen peroxide $\left(\mathrm{H}_{2} \mathrm{O}_{2}\right)$ to yield estolide with hydroxyl groups, which were then end-capped with various organic acids of different chain lengths and structures, while the carboxylic acid group of estolide was esterified with 2-ethylhexanol (2EH). Analysis results revealed that products end-capped with lauric acid (C12) and 2-ethylhexanoic acid, respectively recorded the best pour point at $-36^{\circ} \mathrm{C}$, while other products made with shorter or longer chain length organic acids showed higher pour points. Additionally, product end-capped with longer chain length organic acid exhibited better anti-wear properties and lower kinematic viscosity than product made with shorter chain length organic acid. Furthermore, the oxidation stability of products end-capped with linear organic acid were higher than those end-capped with branched organic acids and the oxidation onset temperature (OOT) of products were between $193^{\circ} \mathrm{C}-200^{\circ} \mathrm{C}$, which is an improvement in comparison with starting material. Physicochemical properties evaluation revealed that most of the prepared estolides esters have lubricant properties comparable to commercial product, which suggested that they are suitable to be used as base oil for lubricants.
\end{abstract}

\section{Keywords: epoxidation, esterification, estolide, fatty acids, lubricant.}

Received: 25 February 2021; Accepted: 24 May 2021; Published online: 21 July 2021.

\section{INTRODUCTION}

Most of lubricants that we use today are made with non-renewable petrochemicals, which have poor biodegradability and consequently are harmful to the environment. These undesirable traits of petrochemical-based lubricants make them a great threat to the environment because of their disposal and inadvertent release to the environment (Nowak et al., 2019; Luther, 2007). As a countermeasure to this problem, some countries have implemented regulations that favour the use of biolubricants such as the 2002 Farm Bill (Farm Security and Rural Investment Act) (Erhan et al., 2006), California Senate

\footnotetext{
Malaysian Palm Oil Board,

6 Persiaran Institusi, Bandar Baru Bangi,

43000 Kajang, Selangor, Malaysia.

E-mail: sengsoi@mpob.gov.my
}

Bill 916 introduced in 2014 (Abi-Akar, 2017) and a Belgium law that imposes the use of biolubricants in all operations conducted near non-navigable waters (Salimon et al., 2010).

Additionally, the German government had supported the use of biolubricants by implementation of 'Market Introduction Program' for biolubricants from year 2001 to 2008, in which financial grants, and technical advices were given to companies that adopted biolubricants (Theissen, 2010). Furthermore, current global consumers and lubricant industry players are shifting towards environment friendly lubricants by adopting biolubricants (Reeves et al., 2017; Cecilia et al., 2020). All these 'green' demand and initiatives have contributed to steadily increasing production of biolubricants as much as $10 \%$ annually (Chan et al., 2018). Based on current development trend for lubricant market, the future of biolubricant is very promising. 
Biolubricants have been made from plant oils and animal fats such as palm oil, soyabean oil, rapeseed oil and tallow, which are sustainable and renewable resources. Plant oil-based biolubricants are known to be environmental-friendly because of their excellent biodegradability as compared to petrochemical-based lubricants (Parveez et al., 2020; Borugadda and Dalai, 2018; Battersby et al., 1992). Additionally, they also offer other beneficial properties such as low volatility, high flash point and high viscosity indices (Sammaiah et al., 2016; Santos et al., 2004). Furthermore, the polar ester group of plant oil-based biolubricant contributes to good boundary lubrication because of its attraction towards metal surface (Salimon et al., 2010) and better solubility for polar contaminant as well as polar additives (Erhan et al., 2006).

Nonetheless, plant oil-based biolubricant is not without drawbacks due to its triglycerides molecular structure. One of the main drawbacks is poor oxidation stability mainly because of the alkene group of unsaturated fatty acids that is susceptible to oxidation through free radical mechanism. Consequently, the viscosity of plant oil-based biolubricant will increase over time and form insoluble deposits that shorten the service lifespan of the biolubricant (Nowicki et al., 2019; Soni and Agarwal, 2014). Another major weakness of plant oil-based biolubricant is the high melting point attribute of plant oil that translates to high pour point for corresponding biolubricant, which limits its usage in cold climate applications. As such, the plant oil-based biolubricants have high tendency to solidify in winter climate that make them unfavourable to consumer (Kim et al., 2019; Kassfeldt and Goran, 1997).

The disadvantages of plant oil-based biolubricant can be resolved by chemical modification of the alkene group of plant oil or its derivatives such as fatty acids and methyl esters. One of the main methods to modify alkene group of unsaturated plant oil derivatives is through epoxidation of alkene with hydrogen peroxide $\left(\mathrm{H}_{2} \mathrm{O}_{2}\right)$ and formic acid to generate epoxidised plant oil derivatives (Chen et al., 2019; Hoong et al., 2008; Armylisas et al., 2017). The purified epoxidised plant oil derivatives were then subjected to epoxide-ring opening reaction with various nucleophiles such as fatty acids (Salih et al., 2011), alcohols (Salimon et al., 2011) and water (Lv et al., 2017; Adhvaryu et al., 2005) to yield modified plant oil derivatives. Subsequent reactions conducted to convert all functional groups of modified plant oil derivatives to ester functionality, significantly lower the pour point and improved the oxidation stability of plant oil-based biolubricants. Nevertheless, the main drawback of this method is the multi-steps process including purification steps required to produce biolubricants with commercially acceptable properties, which contribute to high production cost that may not be economically attractive to consumer.

Alternatively, the properties of plant oil-based lubricants can be improved through a simpler method known as transesterification, in which plant oils can be reacted with various polyhydric alcohols to generate polyol esters that exhibited improved lubricant properties. For example, Heikel et al. (2017) reported a two-steps synthesis of trimethylolpropane (TMP) esters from palm oil and jatropha oil. Both plant oils were first converted to methyl esters through transesterification with methanol and subsequently the methyl esters were further transesterified with TMP to yield TMP esters that exhibited improved cold flow properties in comparison with starting materials. Additionally, the prepared TMP esters also showed better viscosity indices and improved flash points. However, the prepared TMP esters still contain alkene groups that are prone to oxidation degradation, which could reduce the service life of the lubricants. Hence, it is beneficial to make biolubricants with minimal amount of alkene group and simultaneously display good cold flow properties.

Another renowned approach to improve the lubricant properties of plant oil-based lubricant is through formation of estolide from unsaturated fatty acids that generally show good cold flow properties with minimal alkene group in resultant biolubricants (Chen et al., 2020). Estolide is formed when the carboxylic acid group of a fatty acid reacts with the alkene group of another unsaturated fatty acid to form an ester linkage between the two fatty acids, in which the reaction requires the use of strong acids such as perchloric acid as the catalyst (Cermak and Isbell, 2001). Further reaction of the estolide with a branched alcohol afforded estolide ester that achieved desirable low pour point (Cermak et al., 2015; Cermak and Isbell, 2003a; 2003b). Nonetheless, the disadvantage of this approach is the need to use corrosive and hazardous perchloric acid that is known to form explosive mixture, which poses significant threats to human and the environment (Everett and Graf, 1971). Therefore, an alternative method to make estolide without the use of perchloric acid would be a way forward.

An alternative method to make estolide is through esterification of hydroxy fatty acid such as ricinoleic acid with other fatty acids (Salih $e$ t al., 2013) or diacids (Sammaiah et al., 2016). Similarly, the prepared estolide esters originated from hydroxy fatty acids displayed excellent cold flow properties and good thermal oxidation stability. Nonetheless, the main drawback of this approach is that the prepared estolides still contain significant amount of alkene groups that require further chemical modification in order to improve the oxidation stability of the prepared estolides, which will incur 
higher production cost that may hinder commercial acceptance (Greco-Duarte et al., 2019; Cermak et al., 2006). Additionally, global production of hydroxy fatty acids is substantially less than commodity fatty acid and the cost of hydroxy fatty acid is significantly higher than commodity fatty acid such as oleic acid (Mubofu, 2016), which makes hydroxy fatty acid less attractive to be used as raw material for estolide-based biolubricant.

After taking into consideration the advantages and weaknesses of disclosed methods for chemical modification of plant oils and fatty esters, we have reported a direct one-pot method to synthesis polyhydroxy estolide (PE) from oleic acid (Hoong et al., 2017). The reported method did not require the use of purified epoxidised methyl esters or hydroxy fatty acids as starting material, which simplify the process to produce estolides. The process also did not use hazardous perchloric acid as catalyst, instead it used only $\mathrm{H}_{2} \mathrm{O}_{2}$ as the reactant. Additionally, the prepared PE had minimal alkene group that imparted good oxidation stability to lubricant made from it. Nevertheless, the prepared PE has multiple hydroxyl groups that need to be converted to ester group in order to further enhance its oxidation stability and low temperature properties.

A thorough search of relevant literature revealed that no previous study has investigated the structure-properties relationship between organic acids employed to end-cap the hydroxyl groups of PE and the physicochemical properties of the resultant estolide esters. Therefore, the objective of this study is to prepare various organic acids endcapped PE and their respective 2-ethylhexyl estolide esters. Another objective is to examine the structureproperties relationship between the end-capping organic acids and the physicochemical properties of the prepared estolide esters. The findings of this study will help to establish the aptness of estolide esters made from organic acids end-capped PE as lubricant base oil.

\section{MATERIALS AND METHODS}

\section{Materials}

Oleic acid (75\%, PALMAC 750) was kindly provided by IOI Oleochemical Industries Bhd. (Pulau Pinang, Malaysia). $\mathrm{H}_{2} \mathrm{O}_{2}\left(50 \%\right.$ in $\left.\mathrm{H}_{2} \mathrm{O}\right)$, acetic anhydride (99\%), isovaleric acid (bC5) (98\%), hexanoic acid (C6) (98\%), octanoic acid (C8) (99\%), lauric acid (C12) (98\%), stearic acid (C18) (95\%), 2-ethylhexanoic acid (bC8) (99\%) and 2-ethylhexanol (2EH) $(99 \%)$ were purchased from Sigma Aldrich (St. Louis, MO, USA). Pentaerythritol tetraoleate was obtained from commercial sources. All chemicals were used as received without further purification.

\section{Characterisation and Procedures}

Fourier-transform infrared spectroscopy (FTIR) spectra of products were recorded neat on a PerkinElmer FTIR Spectrum 100 with 16 scans per sample at $4 \mathrm{~cm}^{-1}$ resolution in the range of $650-4000 \mathrm{~cm}^{-1}$.

Proton $\left({ }^{1} \mathrm{H}\right)$ and carbon $\left({ }^{13} \mathrm{C}\right)$ nuclear magnetic resonance (NMR) spectroscopy were recorded using JOEL JNM-ECZ600R at $600 \mathrm{MHz}$ and $150 \mathrm{MHz}$, respectively. Sample $\left(10 \mathrm{mg} \mathrm{ml}^{-1}\right)$ was prepared with deuterated chloroform.

Gel permeation chromatography (GPC) was carried out using Polymer Laboratories PL-GPC 50 Plus integrated GPC system furnished with differential refractive index (DRI)/viscometer detectors. Sample (2 $\left.\mathrm{mg} \mathrm{ml}^{-1}\right)$ was dissolved in tetrahydrofuran (THF) and was analysed through a set of Phenogel columns with molecular weight in the range of $10^{2}-10^{6} \mathrm{Da}$. Sample was eluted in THF at a flow rate of $1 \mathrm{ml} \mathrm{min} \mathrm{m}^{-1}$. The GPC was calibrated using polystyrene standards with range from 162 to $1 \times 10^{5}$ Da.

Wet chemistry analysis was conducted in accordance with American Oil Chemists' Society (AOCS) methods: Acid value (AV) Te 2a-64; Hydroxyl value (OHV) Cd 13-60; Iodine value (IV) Cd 1d-92; Oxirane oxygen content (OOC) Cd 9-57.

Kinematic viscosity measurement was carried out according to ASTM D445 in calibrated Ubbellohde tubes submerged in a Normalab NVB Classic viscosity bath. Viscosity index (VI) was calculated in accordance with American Society for Testing and Materials (ASTM) - ASTM D2270-93.

Pour point of sample was recorded using a Normalab CPP Classic test cabinet with integrated cooling system in accordance with ASTM D97.

The oxidation onset temperature (OOT) of sample was determined using DSC Q20P thermal analyser (TA Instruments) in accordance with ASTM E2009.

The anti-wear property of sample was assessed through wear scar diameter (WSD) test in accordance with ASTM 4172 (Four-ball method) by using a TR30L four-ball equipment (DUCOM Instrument). Steel balls were acquired from SKF Malaysia Sdn. Bhd.

\section{Synthesis of PE from Oleic Acid}

Oleic acid (300 g, 1.06 mole) and $\mathrm{H}_{2} \mathrm{O}_{2}(50 \%$ in $\left.\mathrm{H}_{2} \mathrm{O}\right)(360 \mathrm{~g}, 5.3$ mole) were weighed into a 1 litre three-neck round bottom flask equipped with a condenser, thermometer and magnetic stirrer. The mixture was stirred (500 rpm) and heated at $80^{\circ} \mathrm{C}$ for $24 \mathrm{hr}$. Then, reaction mixture was poured into a separating funnel and left to separate. Next, the aqueous layer was discharged from the organic layer. Subsequently, ethyl acetate $(200 \mathrm{ml})$ and water $(200 \mathrm{ml})$ were added to the organic layer. Again, the 
aqueous layer was discharged from the separating funnel. The organic layer was washed with more water $(2 \times 200 \mathrm{ml})$. The organic layer was dried with anhydrous magnesium sulphate $\left(\mathrm{MgSO}_{4}\right)$ and ethyl acetate was removed to yield a viscous colourless liquid labeled as PE (321 g, 76\% yield). $\mathrm{v}_{\max } / \mathrm{cm}^{-1} 3440(\mathrm{OH}) 2924,2854(\mathrm{C}-\mathrm{H})$ 1733, 1708 $(\mathrm{C}=\mathrm{O}) 1465(\mathrm{C}-\mathrm{H})$ 1241, $1176(\mathrm{C}-\mathrm{O}) 723\left(\mathrm{CH}_{2}\right) ;{ }^{1} \mathrm{H}$ NMR (600 MHz, $\left.\mathrm{CDCl}_{3}\right): \delta_{\mathrm{H}}=4.80-4.76(1 \mathrm{H}, \mathrm{m}$, $\underline{\mathrm{HCOC}}=\mathrm{O}), 3.57-3.52(1 \mathrm{H}, \mathrm{m}, \underline{\mathrm{HCO}}) 3.39-3.34(2 \mathrm{H}$, $\mathrm{m}, \underline{\mathrm{HCO}}), 2.29\left(4 \mathrm{H}, \mathrm{t}, J=7.5 \mathrm{~Hz}, \mathrm{O}=\mathrm{CC}_{2} \mathrm{CH}_{2}\right)$, 1.62-1.53 (4H, m, O=CCH $\left.\mathrm{CH}_{2}\right), 1.46-1.32(8 \mathrm{H}, \mathrm{m}$, $\left.\mathrm{CH}_{2} \mathrm{COH}\right), 1.33-1.17\left(40 \mathrm{H}, \mathrm{m}, \mathrm{CH}_{2} \mathrm{CH}_{2}\right), 0.84(6 \mathrm{H}$, $\left.\mathrm{t}, \mathrm{J}=7.0 \mathrm{~Hz}, \mathrm{CH}_{2} \mathrm{CH}_{3}\right) ;{ }^{13} \mathrm{C}$ NMR $\left(150 \mathrm{MHz}^{2} \mathrm{CDCl}_{3}\right)$ : $\delta_{\mathrm{C}}=179.0,174.0(\mathrm{C}=\mathrm{O}), 76.3(\mathrm{HC} O \mathrm{C}=\mathrm{O}), 74.6,72.5$ (Hㅁ-O), $34.5\left(\mathrm{O}=\mathrm{CCH}_{2}\right), 25.1\left(\mathrm{O}=\mathrm{CCH}_{2} \underline{\mathrm{CH}}_{2}\right), 31.9$, 29.4, 24.7, $22.7\left(\mathrm{CH}_{2} \underline{\mathrm{C}} \mathrm{H}_{2}\right), 14.2\left(\mathrm{CH}_{2} \underline{\mathrm{CH}}{ }_{3}\right)$.

\section{Synthesis of Acetylated Estolide (C2E)}

$\mathrm{PE}(230 \mathrm{~g}, 0.70$ mole of $\mathrm{OH}, \mathrm{OHV}=170 \mathrm{mg} \mathrm{KOH}$ $\left.\mathrm{g}^{-1}\right)$, acetic anhydride (60 $\mathrm{g}, 0.59$ mole) and xylene $(60 \mathrm{ml})$ were charged into a $500 \mathrm{ml}$ round bottom flask equipped with a Dean-Stark apparatus and magnetic stirrer. The mixture was stirred (300 rpm) and heated to $150^{\circ} \mathrm{C}$ for $16 \mathrm{hr}$. Thereafter, excess acetic anhydride and xylene were distilled from the reaction product at $140^{\circ} \mathrm{C}$ under vacuum of 20 mbar. The product was left to cool to room temperature and chloroform $(200 \mathrm{ml})$ was added to the product. The diluted product was dried with anhydrous $\mathrm{MgSO}_{4}$ and chloroform was removed to yield a viscous yellowish liquid labeled as C2E (229 g, 97\% yield). $\mathrm{v}_{\max } / \mathrm{cm}^{-1} 2925,2855$ (C-H) 1736, $1709(\mathrm{C}=\mathrm{O}) 1464\left(\mathrm{CH}_{2}\right) 1372\left(\mathrm{CH}_{3}\right)$ 1235, $1167(\mathrm{C}-\mathrm{O})$ $723\left(\mathrm{CH}_{2}\right) ;{ }^{1} \mathrm{H} \mathrm{NMR}\left(600 \mathrm{MHz}, \mathrm{CDCl}_{3}\right): \delta_{\mathrm{H}}=4.97-4.92$ $(2 \mathrm{H}, \mathrm{m}, \underline{\mathrm{HCOC}}=\mathrm{O}), 2.3-2.2\left(4 \mathrm{H}, \mathrm{m}, \mathrm{O}=\mathrm{CCH}_{2}\right), 2.02$ $\left(3 \mathrm{H}, \mathrm{s}, \mathrm{O}=\mathrm{CCH}_{3}\right), 1.6-1.52\left(4 \mathrm{H}, \mathrm{m}, \mathrm{O}=\mathrm{CCH}_{2} \mathrm{CH}_{2}\right)$, 1.47-1.41 (4H, m, $\left.\underline{\mathrm{H}}_{2} \mathrm{CHO}\right), 1.32-1.14(44 \mathrm{H}, \mathrm{m}$, $\left.\mathrm{CH}_{2} \mathrm{CH}_{2}\right), 0.81\left(6 \mathrm{H}, \mathrm{t}, J=6.9 \mathrm{~Hz}, \mathrm{CH}_{2} \mathrm{CH}_{3}\right) ;{ }^{13} \mathrm{C} \mathrm{NMR}$ $\left(150 \mathrm{MHz} \mathrm{CDCl}_{3}\right): \delta_{\mathrm{C}}=179.6,173.4,170.7(\mathrm{C}=\mathrm{O})$, 73.9, $73.5(\mathrm{H} \underline{\mathrm{COC}}=\mathrm{O}), 34.3,34.1\left(\mathrm{O}=\mathrm{C} \underline{\mathrm{C}} \mathrm{H}_{2}\right), 25.1$ $\left(\mathrm{O}=\mathrm{CCH}_{2} \mathrm{CH}_{2}\right), 31.8,29.7,29.4,29.2,25.2$ 24.6, 20.9 $\left(\mathrm{CH}_{2} \underline{\mathrm{CH}}_{2}\right), 22.6\left(\mathrm{O}=\mathrm{CCH}_{3}\right), 14.1\left(\mathrm{CH}_{2} \underline{\mathrm{CH}}_{3}\right)$.

\section{General Procedure for Synthesis of Organic Acid- capped Estolide (OAE)}

Synthesis of isovaleric acid-capped estolide (bC5E) as an example. PE (250 g, 0.76 mole of $\mathrm{OH}$, $\mathrm{OHV}=170 \mathrm{mg} \mathrm{KOH} \mathrm{g}^{-1}$ ), bC5 (102 g, 1 mole) were charged into a $500 \mathrm{ml}$ round bottom flask equipped with a Dean-Stark apparatus and magnetic stirrer. The mixture was stirred (300 rpm) and heated to $210^{\circ} \mathrm{C}$ for $8 \mathrm{hr}$. Thereafter, excess bC5 was removed from the reaction product at $200^{\circ} \mathrm{C}$ under vacuum of 20 mbar. The product was left to cool to room temperature and chloroform $(200 \mathrm{ml})$ was added to the product. The diluted product was dried with anhydrous $\mathrm{MgSO}_{4}$ and chloroform was removed to yield a viscous yellowish liquid labeled as bC5E (298 g, 95\% yield). $\mathrm{v}_{\max } / \mathrm{cm}^{-1} 2925,2855$ (CH) 1735, $1709(\mathrm{C}=\mathrm{O}) 1465\left(\mathrm{CH}_{2}\right) 1371\left(\mathrm{CH}_{3}\right)$ 1240, 1166 (C-O) $723\left(\mathrm{CH}_{2}\right) ;{ }^{1} \mathrm{H}$ NMR $\left(600 \mathrm{MHz}, \mathrm{CDCl}_{3}\right)$ : $\delta_{\mathrm{H}}=4.99-4.91(2 \mathrm{H}, \mathrm{m}, \underline{\mathrm{HCOC}}=\mathrm{O}), 2.31-2.26(2 \mathrm{H}, \mathrm{m}$, $\left.\mathrm{O}=\mathrm{CC}_{2}\right), 2.25-2.20\left(2 \mathrm{H}, \mathrm{m}, \mathrm{O}=\mathrm{CC} \underline{H}_{2}\right), 2.18-2.11$ $\left(2 \mathrm{H}, \mathrm{m}, \mathrm{O}=\mathrm{CCH}_{2}\right), 2.08-2.01\left(1 \mathrm{H}, \mathrm{m},\left(\mathrm{CH}_{3}\right)_{2} \mathrm{CH}\right)$, 1.62-1.53 $\left(6 \mathrm{H}, \mathrm{m}, \mathrm{O}=\mathrm{CCH}_{2} \mathrm{CH}_{2}\right), 1.52-1.42(4 \mathrm{H}, \mathrm{m}$, $\left.\mathrm{CH}_{2} \mathrm{CHO}\right), 1.32-1.16\left(44 \mathrm{H}, \mathrm{m}, \mathrm{CH}_{2} \underline{\mathrm{CH}}_{2}\right), 0.93-0.90$ $\left(6 \mathrm{H}, \mathrm{m}, \mathrm{CH}_{3} \mathrm{CH}\right) 0.83\left(6 \mathrm{H}, \mathrm{t}, J=6.0 \mathrm{~Hz}, \mathrm{CH}_{2} \mathrm{CH}_{3}\right)$; ${ }^{13} \mathrm{C}$ NMR $\left(150 \mathrm{MHz} \mathrm{CDCl}_{3}\right): \delta_{\mathrm{C}}=179.7,173.5,172.8$ $(\mathrm{C}=\mathrm{O}), 74.1,73.5(\mathrm{HCOC}=\mathrm{O}), 43.6\left(\mathrm{O}=\mathrm{C}_{\underline{C H}}\right), 34.5$, $34.0\left(\mathrm{O}=\mathrm{C}_{\underline{C H}}\right), 25.7\left(\mathrm{O}=\mathrm{CCH}_{2} \underline{\mathrm{CH}}_{2}\right), 31.9,29.7,29.4$, $29.2,25.5,25.1,24.6\left(\mathrm{CH}_{2} \underline{\mathrm{CH}}_{2}\right), 22.7,22.4\left(\mathrm{CHCH}_{3}\right)$, $14.1\left(\mathrm{CH}_{2} \mathrm{CH}_{3}\right)$.

Synthesis of hexanoic acid-capped estolide (C6E). The experiment was conducted according to the general procedure for synthesis of OAE. PE (250 g, 0.76 mole of $\left.\mathrm{OH}, \mathrm{OHV}=170 \mathrm{mg} \mathrm{KOH} \mathrm{g}^{-1}\right), \mathrm{C} 6$ (116 $\mathrm{g}, 1$ mole), viscous yellowish liquid labeled as $\mathrm{C} 6 \mathrm{E}$ (274 g, 97\% yield). $\mathrm{v}_{\max } / \mathrm{cm}^{-1} 2925,2855$ (C-H) 1735, $1709(\mathrm{C}=\mathrm{O}) 1465\left(\mathrm{CH}_{2}\right) 1377\left(\mathrm{CH}_{3}\right)$ 1242, $1166(\mathrm{C}-\mathrm{O})$ $756\left(\mathrm{CH}_{2}\right) ;{ }^{1} \mathrm{H} \mathrm{NMR}\left(600 \mathrm{MHz}, \mathrm{CDCl}_{3}\right): \delta_{\mathrm{H}}=5.01-4.91$ $(2 \mathrm{H}, \mathrm{m}, \underline{\mathrm{HCOC}}=\mathrm{O}), 2.34-2.28\left(2 \mathrm{H}, \mathrm{m}, \mathrm{O}=\mathrm{CCH}_{2}\right)$, 2.28-2.20 $\left(4 \mathrm{H}, \mathrm{m}, \mathrm{O}=\mathrm{CC} \underline{\mathrm{H}}_{2}\right), 1.64-1.55(6 \mathrm{H}, \mathrm{m}$, $\left.\mathrm{O}=\mathrm{CCH}_{2} \mathrm{CH}_{2}\right), 1.51-1.43\left(4 \mathrm{H}, \mathrm{m}, \mathrm{C}_{2} \mathrm{CHO}\right), 1.35-$ $1.17\left(48 \mathrm{H}, \mathrm{m}, \mathrm{CH}_{2} \underline{\mathrm{CH}}_{2}\right), 0.89-0.83\left(9 \mathrm{H}_{2} \mathrm{~m}, \mathrm{CH}_{3} \mathrm{CH}\right)$; ${ }^{13} \mathrm{C} \mathrm{NMR}\left(150 \mathrm{MHz} \mathrm{CDCl}_{3}\right): \delta_{\mathrm{C}}=179.8,173.5(\mathrm{C}=\mathrm{O})$, 74.0, 73.6 $(\mathrm{HCOC}=\mathrm{O}), 34.4,34.0\left(\mathrm{O}=\mathrm{CC}_{2}\right), 25.5$ $\left(\mathrm{O}=\mathrm{CCH}_{2} \mathrm{CH}_{2}\right), 31.9,31.4,29.7,29.4,29.2,25.2,24.8$, $22.7,22.4\left(\mathrm{CH}_{2} \underline{\mathrm{CH}_{2}}\right), 14.1,13.9\left(\mathrm{CH}_{2} \underline{\mathrm{CH}_{3}}\right)$.

Synthesis of octanoic acid-capped estolide (C8E). The experiment was conducted according to the general procedure for synthesis of OAE. PE (200 g, 0.61 mole of $\mathrm{OH}, \mathrm{OHV}=170 \mathrm{mg} \mathrm{KOH} \mathrm{g}^{-1}$ ), C8 (100 g, 0.69 mole), viscous yellowish liquid labeled as octanoic acid-capped estolide (C8E) (239 g, 98\% yield). $\mathrm{v}_{\max } / \mathrm{cm}^{-1} 2923,2854(\mathrm{C}-\mathrm{H})$ 1735, $1708(\mathrm{C}=\mathrm{O}) 1464\left(\mathrm{CH}_{2}\right) 1377\left(\mathrm{CH}_{3}\right) 1162(\mathrm{C}-\mathrm{O}) 723$ $\left(\mathrm{CH}_{2}\right) ;{ }^{1} \mathrm{H}$ NMR $\left(600 \mathrm{MHz}, \mathrm{CDCl}_{3}\right): \delta_{\mathrm{H}}=4.94-4.86$ $(2 \mathrm{H}, \mathrm{m}, \underline{\mathrm{HCOC}}=\mathrm{O}), 2.27-2.20\left(2 \mathrm{H}, \mathrm{m}, \mathrm{O}=\mathrm{CC}_{2}\right)$, 2.20-2.14 $\left(4 \mathrm{H}, \mathrm{m}, \mathrm{O}=\mathrm{CC} \underline{\mathrm{H}}_{2}\right), 1.58-1.47(6 \mathrm{H}, \mathrm{m}$, $\left.\mathrm{O}=\mathrm{CCH}_{2} \mathrm{CH}_{2}\right), 1.45-1.36\left(4 \mathrm{H}, \mathrm{m}, \mathrm{CH}_{2} \mathrm{CHO}\right), 1.29-$ $1.09\left(52 \mathrm{H}, \mathrm{m}, \mathrm{CH}_{2} \underline{\mathrm{CH}}_{2}\right), 0.81-0.74\left(9 \mathrm{H}, \mathrm{m}, \mathrm{CH}_{3} \mathrm{CH}\right)$; ${ }^{13} \mathrm{C}$ NMR $\left(150 \mathrm{MHz}^{2} \mathrm{CDCl}_{3}\right): \delta_{\mathrm{C}}=179.4,173.3(\mathrm{C}=\mathrm{O})$, 73.8, $73.5(\mathrm{H} \underline{\mathrm{COC}}=\mathrm{O}), 34.4,33.9\left(\mathrm{O}=\mathrm{CCH}_{2}\right), 25.1$ $\left(\mathrm{O}=\mathrm{CCH}_{2} \mathrm{CH}_{2}\right), 31.8,31.6,29.7,29.4,29.2,25.2,24.6$, 22.7, $22.6\left(\mathrm{CH}_{2} \underline{\mathrm{CH}}\right)_{2}, 14.1\left(\mathrm{CH}_{2} \underline{\mathrm{CH}}_{3}\right)$.

Synthesis of 2-ethylhexanoic acid-capped estolide (bC8E). The experiment was conducted according to the general procedure for synthesis of OAE. PE (200 g, 0.61 mole of $\mathrm{OH}, \mathrm{OHV}=170 \mathrm{mg} \mathrm{KOH} \mathrm{g}^{-1}$ ), 2-ethylhexanoic acid (100 g, 0.69 mole), viscous yellowish liquid labeled as 2-ethylhexanoic acid-capped estolide (bC8E) (235 g, 97\% yield). 
$\mathrm{v}_{\max } / \mathrm{cm}^{-1}$ 2925, 2855 (C-H) 1733, 1709 (C=O) $1460\left(\mathrm{CH}_{2}\right) 1377\left(\mathrm{CH}_{3}\right) 1167(\mathrm{C}-\mathrm{O}) 724\left(\mathrm{CH}_{2}\right) ;{ }^{1} \mathrm{H}$ NMR $\left(600 \mathrm{MHz}, \mathrm{CDCl}_{3}\right): \delta_{\mathrm{H}}=5.05-4.88(2 \mathrm{H}, \mathrm{m}$, $\underline{\mathrm{HCOC}}=\mathrm{O}), 2.33-2.29\left(2 \mathrm{H}, \mathrm{m}, \mathrm{O}=\mathrm{CC} \underline{\mathrm{H}}_{2}\right), 2.28-2.17$ $\left(4 \mathrm{H}, \mathrm{m}, \mathrm{O}=\mathrm{CC}_{2}\right), 1.63-1.53\left(8 \mathrm{H}, \mathrm{m}, \mathrm{O}=\mathrm{CCH}_{2} \mathrm{CH}_{2}\right)$, 1.53-1.41 (4H, m, $\left.\mathrm{CH}_{2} \mathrm{CHO}\right), 1.29-1.09(44 \mathrm{H}, \mathrm{m}$, $\left.\mathrm{CH}_{2} \mathrm{CH}_{2}\right), \quad 0.91-0.82\left(12 \mathrm{H}, \mathrm{m}, \mathrm{CH}_{3} \mathrm{CH}\right) ;{ }^{13} \mathrm{C} \mathrm{NMR}$ $\left(150 \mathrm{MHz} \mathrm{CDCl}_{3}\right): \delta_{\mathrm{C}}=179.7,175.9,173.5(\mathrm{C}=\mathrm{O})$, 74.0, $73.5(\mathrm{H} \underline{C O C}=\mathrm{O}), 44.7(\mathrm{H} \underline{C} \mathrm{C}=\mathrm{O}), 34.5,34.1$ $\left(\mathrm{O}=\mathrm{CCH}_{2}\right), 25.6,25.1\left(\mathrm{O}=\mathrm{CCH}_{2} \underline{\mathrm{CH}}{ }_{2}\right), 31.9,31.7$, 29.7, 29.5, 29.2, 25.0, 24.6, 22.7, $22.6\left(\mathrm{CH}_{2} \mathrm{CH}_{2}\right)$, 14.1, $11.9\left(\mathrm{CH}_{2} \underline{\mathrm{CH}_{3}}\right)$.

Synthesis of lauric acid-capped estolide (C12E). The experiment was conducted according to the general procedure for synthesis of OAE. PE (200 g, 0.61 mole of $\left.\mathrm{OH}, \mathrm{OHV}=170 \mathrm{mg} \mathrm{KOH} \mathrm{g}^{-1}\right), \mathrm{C} 12$ (120 $\mathrm{g}, 0.6 \mathrm{~mole})$, viscous yellowish liquid labeled as lauric acid-C12E (247 $\mathrm{g}, 96 \%$ yield $) . \mathrm{v}_{\max } / \mathrm{cm}^{-1} 2923$, $2854(\mathrm{C}-\mathrm{H})$ 1737, $1709(\mathrm{C}=\mathrm{O}) 1465\left(\mathrm{CH}_{2}\right) 1377\left(\mathrm{CH}_{3}\right)$ 1235, 1166 (C-O), $723\left(\mathrm{CH}_{2}\right) ;{ }^{1} \mathrm{H}$ NMR $(600 \mathrm{MHz}$, $\left.\mathrm{CDCl}_{3}\right): \delta_{\mathrm{H}}=5.01-4.92(2 \mathrm{H}, \mathrm{m}, \underline{\mathrm{HCOC}}=\mathrm{O}), 2.32-2.29$ $\left(2 \mathrm{H}, \mathrm{m}, \mathrm{O}=\mathrm{CC} \underline{\mathrm{H}}_{2}\right), 2.28-2.21\left(4 \mathrm{H}, \mathrm{m}, \mathrm{O}=\mathrm{CC} \underline{\mathrm{H}}_{2}\right)$, 1.63-1.54 (6H, m, O=CCH $\left.\mathrm{CH}_{2}\right), 1.52-1.43(4 \mathrm{H}$, $\left.\mathrm{m}, \mathrm{CH}_{2} \mathrm{CHO}\right), 1.33-1.16\left(54 \mathrm{H}, \mathrm{m}, \mathrm{CH}_{2} \underline{\mathrm{CH}}_{2}\right), 0.86-$ $0.82\left(9 \mathrm{H}, \mathrm{m}, \mathrm{CH}_{3} \mathrm{CH}\right) ;{ }^{13} \mathrm{C} \mathrm{NMR}\left(150 \mathrm{MHz} \mathrm{CDCl}_{3}\right)$ : $\delta_{\mathrm{C}}=180.0,173.5(\mathrm{C}=\mathrm{O}), 74.0,73.6(\mathrm{HCOC}=\mathrm{O}), 34.5$, $34.1\left(\mathrm{O}=\mathrm{CCH}_{2}\right), 24.7\left(\mathrm{O}=\mathrm{CCH}_{2} \underline{\mathrm{CH}}_{2}\right), 31.9,29.6,29.4$, 29.2, 25.6, 25.0, $22.7\left(\mathrm{CH}_{2} \underline{\mathrm{CH}_{2}}\right), 14.1\left(\mathrm{CH}_{2} \underline{\mathrm{C}}{ }_{3}\right)$.

Synthesis of stearic acid-capped estolide (C18E). The experiment was conducted according to the general procedure for synthesis of OAE. PE (200 $\mathrm{g}, 0.61$ mole of $\mathrm{OH}, \mathrm{OHV}=170 \mathrm{mg} \mathrm{KOH} \mathrm{g}^{-1}$ ), C18 (100 g, 0.35 mole), viscous yellowish liquid labeled as stearic acid-capped estolide (C18E) (285 g, 98\% yield). $\mathrm{v}_{\max } / \mathrm{cm}^{-1} 2917,2850(\mathrm{C}-\mathrm{H}) 1736,1707(\mathrm{C}=\mathrm{O})$ $1464\left(\mathrm{CH}_{2}\right) 1371\left(\mathrm{CH}_{3}\right)$ 1234, $1166(\mathrm{C}-\mathrm{O}) 721\left(\mathrm{CH}_{2}\right)$; ${ }^{1} \mathrm{H} \mathrm{NMR}\left(600 \mathrm{MHz}, \mathrm{CDCl}_{3}\right): \delta_{\mathrm{H}}=5.02-4.95(2 \mathrm{H}, \mathrm{m}$, $\underline{\mathrm{HCOC}}=\mathrm{O}), 2.35-2.31\left(2 \mathrm{H}, \mathrm{m}, \mathrm{O}=\mathrm{CC}_{2}\right), 2.31-2.23$ $\left(4 \mathrm{H}, \mathrm{m}, \mathrm{O}=\mathrm{CC} \underline{\mathrm{H}}_{2}\right), 1.65-1.57\left(6 \mathrm{H}, \mathrm{m}, \mathrm{O}=\mathrm{CCH}_{2} \underline{\mathrm{H}}_{2}\right)$, 1.53-1.45 (4H, m, $\left.\underline{\mathrm{H}}_{2} \mathrm{CHO}\right), 1.36-1.21(66 \mathrm{H}, \mathrm{m}$, $\left.\mathrm{CH}_{2} \underline{\mathrm{CH}}_{2}\right), 0.87\left(9 \mathrm{H}, \mathrm{t}, \underline{\mathrm{CH}}_{3} \mathrm{CH}\right) ;{ }^{13} \mathrm{C} \mathrm{NMR}(150 \mathrm{MHz}$ $\left.\mathrm{CDCl}_{3}\right): \delta_{\mathrm{C}}=180.1,173.6(\mathrm{C}=\mathrm{O}), 74.1,73.7(\mathrm{HCOC}=\mathrm{O})$, 34.5, 34.1 $\left(\mathrm{O}=\mathrm{CCCH}_{2}\right), 25.2\left(\mathrm{O}=\mathrm{CCH}_{2} \underline{\mathrm{CH}}_{2}\right), 32.0,29.8$, $29.5,29.3,25.6,24.8,22.8\left(\mathrm{CH}_{2} \underline{\mathrm{CH}}_{2}\right), 14.2\left(\mathrm{CH}_{2} \underline{\mathrm{CH}}_{3}\right)$.

\section{General Procedure for Synthesis of 2-ethylhexyl Ester of OAE}

Synthesis of 2-ethylhexyl ester of octanoic acidcapped estolide (2EH-C8E). Octanoic acid-capped estolide (C8E) (182 g, 0.32 mole of $\mathrm{CO}_{2} \mathrm{H}, \mathrm{AV}=$ $100 \mathrm{mg} \mathrm{KOH} \mathrm{g}^{-1}$ ) and 2EH (102 g, 0.78 mole) were weighed into a reaction flask connected to a DeanStark apparatus. The mixture was stirred (300 rpm) and heated at $210^{\circ} \mathrm{C}$ for $8 \mathrm{hr}$. Thereafter, the excess $2 \mathrm{EH}$ was removed through distillation at $210^{\circ} \mathrm{C}$ under 10 mbar vacuum. The product was diluted with $200 \mathrm{ml}$ chloroform and dried with anhydrous $\mathrm{MgSO}_{4}$. Then, solvent was removed to yield a yellowish liquid known as 2-ethylhexyl ester of octanoic acid-capped estolide (2EH-C8E) (215 g, 98\% yield). $\mathrm{v}_{\max } / \mathrm{cm}^{-1} 2924,2855(\mathrm{C}-\mathrm{H}) 1735(\mathrm{C}=\mathrm{O})$ $1463\left(\mathrm{CH}_{2}\right) 1378\left(\mathrm{CH}_{3}\right)$ 1244, $1165(\mathrm{C}-\mathrm{O}) 723\left(\mathrm{CH}_{2}\right)$; ${ }^{1} \mathrm{H} \mathrm{NMR}\left(600 \mathrm{MHz}, \mathrm{CDCl}_{3}\right): \delta_{\mathrm{H}}=4.97-4.87(2 \mathrm{H}, \mathrm{m}$, $\underline{\mathrm{HCOC}}=\mathrm{O}), 3.95-3.88\left(2 \mathrm{H}, \mathrm{m}, \underline{\mathrm{H}}_{2} \mathrm{COC}=\mathrm{O}\right), 2.29-2.16$ $\left(6 \mathrm{H}, \mathrm{m}, \mathrm{O}=\mathrm{CC} \underline{\mathrm{H}}_{2}\right), 1.59-1.51\left(6 \mathrm{H}, \mathrm{m}, \mathrm{O}=\mathrm{CCH}_{2} \mathrm{CH}_{2}\right)$, 1.51-1.46 (1H, m, CHCHO), $1.46-1.40(4 \mathrm{H}, \mathrm{m}$, $\left.\mathrm{CH}_{2} \mathrm{CHO}\right), 1.33-1.10\left(60 \mathrm{H}, \mathrm{m}, \mathrm{CH}_{2} \mathrm{CH}_{2}\right), 0.85-0.76$ $\left(15 \mathrm{H}, \mathrm{m}, \mathrm{CH}_{3} \mathrm{CH}\right) ;{ }^{13} \mathrm{C} \mathrm{NMR}\left(150 \mathrm{MHz} \mathrm{CDCl}_{3}\right)$ : $\delta_{\mathrm{C}}=173.9,173.2(\mathrm{C}=\mathrm{O}), 73.9,73.5(\mathrm{HCOC}=\mathrm{O}), 66.5$ $\left(\mathrm{H}_{2} \underline{\mathrm{COC}}=\mathrm{O}\right) 38.8\left(\mathrm{HCCH}_{2} \mathrm{O}\right) 34.4,34.3\left(\mathrm{O}=\mathrm{CCH}_{2}\right)$, $25.5\left(\mathrm{O}=\mathrm{CCH}_{2} \mathrm{CH}_{2}\right), 31.8,31.7,30.4,29.7,29.4$, 29.3, 28.9, 25.0, 23.8, 22.9, $22.7\left(\mathrm{CH}_{2} \mathrm{CH}_{2}\right), 14.1,10.9$ $\left(\mathrm{CH}_{2} \mathrm{CH}_{3}\right)$.

Synthesis of 2-ethylhexyl ester of acetic acidcapped estolide (2EH-C2E). The experiment was conducted according to the general procedure for synthesis of 2-ethylhexyl ester of OAE. Acetic acidcapped estolide (C2E) (195 g, 0.27 mole of $\mathrm{CO}_{2} \mathrm{H}$, $\mathrm{AV}=80 \mathrm{mg} \mathrm{KOH} \mathrm{g}^{-1}$ ) and $2 \mathrm{EH}$ (75 g, 0.57 mole). A yellowish liquid known as 2-ethylhexyl ester of acetic acid-capped estolide (2EH-C2E) (224 g, $98 \%$ yield ). $\mathrm{v}_{\max } / \mathrm{cm}^{-1} 2925,2855(\mathrm{C}-\mathrm{H}) 1735(\mathrm{C}=\mathrm{O})$ $1463\left(\mathrm{CH}_{2}\right) 1373\left(\mathrm{CH}_{3}\right)$ 1234, $1169(\mathrm{C}-\mathrm{O}) 724\left(\mathrm{CH}_{2}\right)$; ${ }^{1} \mathrm{H}$ NMR $\left(600 \mathrm{MHz}, \mathrm{CDCl}_{3}\right): \delta_{\mathrm{H}}=4.95-4.87(2 \mathrm{H}, \mathrm{m}$, $\underline{\mathrm{HCOC}}=\mathrm{O}), 3.93-3.85\left(2 \mathrm{H}, \mathrm{m}, \underline{\mathrm{H}}_{2} \mathrm{COC}=\mathrm{O}\right), 2.26-2.15$ $\left(4 \mathrm{H}, \mathrm{m}, \mathrm{O}=\mathrm{CC} \underline{\mathrm{H}}_{2}\right), 1.97\left(3 \mathrm{H}, \mathrm{s}, \mathrm{O}=\mathrm{CCH}_{3}\right)$ 1.56-1.49 $\left(4 \mathrm{H}, \mathrm{m}, \mathrm{O}=\mathrm{CCH}_{2} \underline{\mathrm{CH}}_{2}\right), 1.49-1.44(1 \mathrm{H}, \mathrm{m}, \mathrm{C} \underline{\mathrm{HCHO}})$, 1.43-1.37 (4H, m, $\left.\underline{\mathrm{H}}_{2} \mathrm{CHO}\right), 1.33-1.10(52 \mathrm{H}, \mathrm{m}$, $\left.\mathrm{CH}_{2} \mathrm{CH}_{2}\right), 0.84-0.73\left(12 \mathrm{H}, \mathrm{m}, \mathrm{CH}_{3} \mathrm{CH}\right) ;{ }^{13} \mathrm{C} \mathrm{NMR}(150$ $\left.\mathrm{MHz} \mathrm{CDCl}_{3}\right): \delta_{\mathrm{C}}=173.9,173.2,170.4(\mathrm{C}=\mathrm{O}), 73.8,73.4$ $(\mathrm{H} \underline{C O C}=\mathrm{O}), 66.5\left(\mathrm{H}_{2} \underline{\mathrm{COC}}=\mathrm{O}\right) 38.7\left(\mathrm{HCCH}_{2} \mathrm{O}\right) 34.3$ $\left(\mathrm{O}=\mathrm{CCH}_{2}\right), 25.4\left(\mathrm{O}=\mathrm{CCH}_{2} \underline{C H}_{2}\right), 31.8,30.4,29.6,29.3$, $28.9,25.0,23.8,22.9,22.6\left(\mathrm{CH}_{2} \underline{\mathrm{CH}}_{2}\right), 20.9\left(\mathrm{H}_{3} \underline{\mathrm{CC}}=\mathrm{O}\right)$ $14.1\left(\mathrm{CH}_{2} \mathrm{CH}_{3}\right)$.

Synthesis of 2-ethylhexyl ester of isovaleric acidcapped estolide (2EH-bC5E). The experiment was conducted according to the general procedure for synthesis of 2-ethylhexyl ester of OAE. bC5E (200 g, 0.31 mole of $\mathrm{CO}_{2} \mathrm{H}, \mathrm{AV}=86 \mathrm{mg} \mathrm{KOH} \mathrm{g}^{-1}$ ) and 2EH (120 g, 0.92 mole). A yellowish liquid known as 2EH-bC5E (224 g, 96\% yield). $\mathrm{v}_{\max } / \mathrm{cm}^{-1} 2925$, $2855(\mathrm{C}-\mathrm{H}) 1735(\mathrm{C}=\mathrm{O}) 1464\left(\mathrm{CH}_{2}\right) 1370\left(\mathrm{CH}_{3}\right)$ 1245, 1167 (C-O) $724\left(\mathrm{CH}_{2}\right) ;{ }^{1} \mathrm{H}$ NMR $\left(600 \mathrm{MHz}, \mathrm{CDCl}_{3}\right)$ : $\delta_{\mathrm{H}}=4.99-4.90(2 \mathrm{H}, \mathrm{m}, \underline{\mathrm{HCOC}}=\mathrm{O}), 3.97-3.90(2 \mathrm{H}, \mathrm{m}$, $\left.\underline{\mathrm{H}}_{2} \mathrm{COC}=\mathrm{O}\right), 2.29-2.20\left(4 \mathrm{H}, \mathrm{m}, \mathrm{O}=\mathrm{CC}_{2}\right), 2.19-2.11$ $\left(2 \mathrm{H}, \mathrm{m}, \mathrm{O}=\mathrm{CC} \underline{\mathrm{H}}_{2}\right), 2.10-2.01\left(1 \mathrm{H}, \mathrm{m},\left(\mathrm{CH}_{3}\right)_{2} \mathrm{CH}\right)$, 1.61-1.54 (4H, m, O=CCH$\left.{ }_{2} \underline{\mathrm{H}}_{2}\right), 1.53-1.49(1 \mathrm{H}, \mathrm{m}$, C $\underline{\mathrm{HCHO}}), 1.49-1.42\left(4 \mathrm{H}, \mathrm{m}, \mathrm{CH}_{2} \mathrm{CHO}\right), 1.37-1.15$ $\left(52 \mathrm{H}, \mathrm{m}, \mathrm{CH}_{2} \mathrm{CH}_{2}\right), 0.94-0.89\left(6 \mathrm{H}, \mathrm{m}, \mathrm{HCCH}_{3}\right)$, 0.88-0.80 (12H, m, $\left.\mathrm{CH}_{3} \mathrm{CH}\right) ;{ }^{13} \mathrm{C} \mathrm{NMR} \mathrm{(150} \mathrm{MHz}$ $\left.\mathrm{CDCl}_{3}\right): \delta_{\mathrm{C}}=173.9,173.3,172.6(\mathrm{C}=\mathrm{O}), 74.0,73.8$ $(\mathrm{HCOC}=\mathrm{O}), \quad 66.6 \quad\left(\mathrm{H}_{2} \underline{\mathrm{COC}}=\mathrm{O}\right) \quad 43.6 \quad\left(\mathrm{O}=\mathrm{C}_{\underline{C H}}\right)$, $38.8\left(\mathrm{HCCH}_{2} \mathrm{O}\right), \quad 34.5, \quad 34.4 \quad\left(\mathrm{O}=\mathrm{CLCH}_{2}\right), \quad 25.7$ 
$\left(\mathrm{O}=\mathrm{CCH}_{2} \mathrm{CH}_{2}\right), 31.8,30.5,29.7,29.4,29.2,28.9,25.0$, 23.8, $\left.23.0\left(\mathrm{CH}_{2} \underline{\mathrm{CH}}_{2}\right), 22.7,22.4\left(\mathrm{CH}_{\mathrm{CH}}\right)_{3}\right), 14.1,11.0$ $\left(\mathrm{CH}_{2} \mathrm{CH}_{3}\right)$.

Synthesis of 2-ethylhexyl ester of hexanoic acidcapped estolide (2EH-C6E). The experiment was conducted according to the general procedure for synthesis of 2-ethylhexyl ester of OAE. C6E (200 g, 0.32 mole of $\left.\mathrm{CO}_{2} \mathrm{H}, \mathrm{AV}=90 \mathrm{mg} \mathrm{KOH} \mathrm{g}^{-1}\right)$ and $2 \mathrm{EH}$ (100 g, 0.77 mole). A yellowish liquid known as 2-ethylhexyl ester of hexanoic acid-capped estolide (2EH-C6E) (232 g, 98\% yield). $\mathrm{v}_{\max } / \mathrm{cm}^{-1} 2925,2856$ (C-H) $1734(\mathrm{C}=\mathrm{O}) 1463\left(\mathrm{CH}_{2}\right)^{\max } 1378\left(\mathrm{CH}_{3}\right)$ 1242, 1168 (C-O) $725\left(\mathrm{CH}_{2}\right) ;{ }^{1} \mathrm{H}$ NMR (600 MHz, $\left.\mathrm{CDCl}_{3}\right)$ : $\delta_{\mathrm{H}}=4.99-4.90(2 \mathrm{H}, \mathrm{m}, \underline{\mathrm{HCOC}}=\mathrm{O}), 3.98-3.91(2 \mathrm{H}, \mathrm{m}$, $\left.\underline{\mathrm{H}}_{2} \mathrm{COC}=\mathrm{O}\right), 2.32-2.21\left(6 \mathrm{H}, \mathrm{m}, \mathrm{O}=\mathrm{CCH}_{2}\right), 1.63-1.55$ $\left(6 \mathrm{H}, \mathrm{m}, \mathrm{O}=\mathrm{CCH}_{2} \mathrm{CH}_{2}\right), 1.55-1.49(1 \mathrm{H}, \mathrm{m}, \mathrm{C} \underline{\mathrm{HCHO}})$, 1.49-1.43 (4H, m, $\left.\underline{\mathrm{C}}_{2} \mathrm{CHO}\right), 1.37-1.17(56 \mathrm{H}, \mathrm{m}$, $\left.\mathrm{CH}_{2} \mathrm{CH}_{2}\right), \quad 0.89-0.82\left(15 \mathrm{H}, \mathrm{m}, \mathrm{CH}_{3} \mathrm{CH}\right) ;{ }^{13} \mathrm{C} \mathrm{NMR}$ $\left(150 \mathrm{MHz} \mathrm{CDCl}_{3}\right): \delta_{\mathrm{C}}=174.0,173.4(\mathrm{C}=\mathrm{O}), 73.9$, $73.5(\mathrm{HCOC}=\mathrm{O}), 66.7\left(\mathrm{H}_{2} \underline{\mathrm{COC}}=\mathrm{O}\right) 38.8\left(\mathrm{HCCH}_{2} \mathrm{O}\right)$ $34.5\left(\mathrm{O}=\mathrm{CCH}_{2}\right), 25.5\left(\mathrm{O}=\mathrm{CCH}_{2} \underline{\mathrm{CH}}_{2}\right), 31.8,31.4,30.5$, 29.7, 29.4, 29.2, 28.9, 25.2, 25.0, 24.8, 23.8, 23.0, 22.7 $\left(\mathrm{CH}_{2} \underline{\mathrm{CH}}_{2}\right), 14.1,11.0\left(\mathrm{CH}_{2} \underline{\mathrm{CH}}_{3}\right)$.

Synthesis of 2-ethylhexyl ester of 2-ethylhexanoic acid-capped estolide (2EH-bC8E). The experiment was conducted according to the general procedure for synthesis of 2-ethylhexyl ester of OAE. 2-ethylhexanoic acid-capped estolide (bC8E) (188 $\mathrm{g}, 0.26$ mole of $\mathrm{CO}_{2} \mathrm{H}, \mathrm{AV}=79 \mathrm{mg} \mathrm{KOH} \mathrm{g}^{-1}$ ) and 2EH (70 g, 0.54 mole). A yellowish liquid known as 2-ethylhexyl ester of 2-ethylhexanoic acid-capped estolide (2EH-bC8E) (210 g, 97\% yield). $\mathrm{v}_{\max } / \mathrm{cm}^{-1}$ 2925, $2856(\mathrm{C}-\mathrm{H}) 1734(\mathrm{C}=\mathrm{O}) 1464\left(\mathrm{CH}_{2}\right) 1378\left(\mathrm{CH}_{3}\right)$ 1238, 1167 (C-O) $724\left(\mathrm{CH}_{2}\right)$; ${ }^{1} \mathrm{H}$ NMR $(600 \mathrm{MHz}$, $\left.\mathrm{CDCl}_{3}\right): \delta_{\mathrm{H}}=5.04-4.86(2 \mathrm{H}, \mathrm{m}, \underline{\mathrm{HCOC}}=\mathrm{O}), 3.97-3.90$ $\left(2 \mathrm{H}, \mathrm{m}, \underline{\mathrm{H}}_{2} \mathrm{COC}=\mathrm{O}\right), 2.30-2.17\left(6 \mathrm{H}, \mathrm{m}, \mathrm{O}=\mathrm{CC}_{2}\right)$, 1.63-1.53 $\left(8 \mathrm{H}, \mathrm{m}, \mathrm{O}=\mathrm{CCH}_{2} \underline{\mathrm{CH}}_{2}\right), 1.52-1.49(1 \mathrm{H}, \mathrm{m}$, $\mathrm{C} \underline{\mathrm{HCHO}}), 1.48-1.42\left(4 \mathrm{H}, \mathrm{m}, \mathrm{C}_{2} \mathrm{CHO}\right), 1.35-1.12$ $\left(56 \mathrm{H}, \mathrm{m}, \mathrm{CH}_{2} \mathrm{CH}_{2}\right), 0.89-0.79\left(18 \mathrm{H}, \mathrm{m}, \mathrm{CH}_{3} \mathrm{CH}\right) ;{ }^{13} \mathrm{C}$ $\operatorname{NMR}\left(150 \mathrm{MHz} \mathrm{CDCl}_{3}\right): \delta_{\mathrm{C}}=175.8,173.9(\mathrm{C}=\mathrm{O}), 74.2$, $73.9(\mathrm{HCOC}=\mathrm{O}), 66.2\left(\mathrm{H}_{2} \underline{\mathrm{COC}}=\mathrm{O}\right) 47.7(\mathrm{HCC}=\mathrm{O})$, $38.8\left(\mathrm{HCCH}_{2} \mathrm{O}\right) 34.4\left(\mathrm{O}=\mathrm{CCH}_{2}\right), 25.5\left(\mathrm{O}=\mathrm{CCH}_{2} \mathrm{CH}_{2}\right)$, $31.9,30.5,29.7,29.4,29.2,28.9,25.0,23.8,23.0,22.7$ $\left(\mathrm{CH}_{2} \underline{\mathrm{CH}}_{2}\right), 14.1,11.8,11.0\left(\mathrm{CH}_{2} \underline{\mathrm{CH}}_{3}\right)$.

Synthesis of 2-ethylhexyl ester of lauric acidcapped estolide (2EH-C12E). The experiment was conducted according to the general procedure for synthesis of 2-ethylhexyl ester of OAE. Lauric acidcapped estolide (C12E) (200 g, 0.37 mole of $\mathrm{CO}_{2} \mathrm{H}$, $\mathrm{AV}=103 \mathrm{mg} \mathrm{KOH} \mathrm{g}^{-1}$ ) and 2EH (96 g, 0.74 mole). A yellowish liquid known as 2-ethylhexyl ester of lauric acid-capped estolide (2EH-C12E) (235 g, 97\% yield). $\mathrm{v}_{\max } / \mathrm{cm}^{-1} 2924,2855(\mathrm{C}-\mathrm{H}) 1736(\mathrm{C}=\mathrm{O})$ $1464\left(\mathrm{CH}_{2}\right) 1378\left(\mathrm{CH}_{3}\right)$ 1236, $1169(\mathrm{C}-\mathrm{O}) 724\left(\mathrm{CH}_{2}\right)$; ${ }^{1} \mathrm{H} \mathrm{NMR}\left(600 \mathrm{MHz}, \mathrm{CDCl}_{3}\right): \delta_{\mathrm{H}}=4.98-4.91(2 \mathrm{H}, \mathrm{m}$, $\underline{\mathrm{HCOC}}=\mathrm{O}), 3.96-3.89\left(2 \mathrm{H}, \mathrm{m}, \underline{\mathrm{H}}_{2} \mathrm{COC}=\mathrm{O}\right), 2.30-2.18$ $\left(6 \mathrm{H}, \mathrm{m}, \mathrm{O}=\mathrm{CC}_{2}\right), 1.63-1.53\left(6 \mathrm{H}, \mathrm{m}, \mathrm{O}=\mathrm{CCH}_{2} \mathrm{CH}_{2}\right)$, 1.52-1.47 (1H, m, C $\left.\mathrm{CH}_{2} \mathrm{CHO}\right), 1.35-1.11\left(66 \mathrm{H}, \mathrm{m}, \mathrm{CH}_{2} \underline{\mathrm{CH}}_{2}\right), 0.88-0.78$ $\left(15 \mathrm{H}, \mathrm{m}, \mathrm{CH}_{3} \mathrm{CH}\right) ;{ }^{13} \mathrm{C} \mathrm{NMR}\left(150 \mathrm{MHz} \mathrm{CDCl}_{3}\right)$ : $\delta_{\mathrm{C}}=174.0,173.3(\mathrm{C}=\mathrm{O}), 73.9,73.5(\mathrm{HCOC}=\mathrm{O}), 66.6$ $\left(\mathrm{H}_{2} \underline{\mathrm{COC}}=\mathrm{O}\right) 38.8\left(\mathrm{HCCH}_{2} \mathrm{O}\right) 34.5,34.3\left(\mathrm{O}=\mathrm{C}_{2} \mathrm{H}_{2}\right)$, $25.5\left(\mathrm{O}=\mathrm{CCH}_{2} \mathrm{CH}_{2}\right), 31.9,31.7,30.5,29.6,29.4$, 29.2, 28.9, 25.1, 23.8, 23.0, $22.7\left(\mathrm{CH}_{2} \underline{\mathrm{CH}}_{2}\right), 14.1,11.0$ $\left(\mathrm{CH}_{2} \mathrm{CH}_{3}\right)$.

Synthesis of 2-ethylhexyl ester of stearic acidcapped estolide (2EH-C18E). The experiment was conducted according to the general procedure for synthesis of 2-ethylhexyl ester of OAE. Stearic acid-capped estolide (C18E) (200 g, 0.35 mole of $\left.\mathrm{CO}_{2} \mathrm{H}, \mathrm{AV}=99 \mathrm{mg} \mathrm{KOH} \mathrm{g}{ }^{-1}\right)$ and $2 \mathrm{EH}(92 \mathrm{~g}, 0.71$ mole). A yellowish liquid known as 2-ethylhexyl ester of stearic acid-capped estolide (2EH-C18E) (232 g, 97\% yield). $v_{\max } / \mathrm{cm}^{-1} 2923,2854(\mathrm{C}-\mathrm{H})$ $1736(\mathrm{C}=\mathrm{O}) 1464\left(\mathrm{CH}_{2}\right) 1378\left(\mathrm{CH}_{3}\right)$ 1242, 1169 (C-O) $723\left(\mathrm{CH}_{2}\right) ;{ }^{1} \mathrm{H}$ NMR $\left(600 \mathrm{MHz}, \mathrm{CDCl}_{3}\right)$ : $\delta_{\mathrm{H}}=5.03-4.91(2 \mathrm{H}, \mathrm{m}, \underline{\mathrm{HCOC}}=\mathrm{O}), 3.99-3.91(2 \mathrm{H}, \mathrm{m}$, $\left.\underline{\mathrm{H}}_{2} \mathrm{COC}=\mathrm{O}\right), 2.32-2.21\left(6 \mathrm{H}, \mathrm{m}, \mathrm{O}=\mathrm{CC} \underline{\mathrm{H}}_{2}\right), 1.61-1.56$ $\left(6 \mathrm{H}, \mathrm{m}, \mathrm{O}=\mathrm{CCH}_{2} \underline{\mathrm{CH}}_{2}\right), 1.56-1.51(1 \mathrm{H}, \mathrm{m}, \mathrm{C} \underline{\mathrm{HCHO}})$, 1.49-1.44 (4H, m, $\left.\underline{\mathrm{CH}}_{2} \mathrm{CHO}\right), 1.35-1.18(80 \mathrm{H}, \mathrm{m}$, $\left.\mathrm{CH}_{2} \mathrm{CH}_{2}\right), \quad 0.89-0.81\left(15 \mathrm{H}, \mathrm{m}, \mathrm{CH}_{3} \mathrm{CH}\right) ;{ }^{13} \mathrm{C}$ NMR $\left(150 \mathrm{MHz} \mathrm{CDCl}_{3}\right): \delta_{\mathrm{C}}=174.0,173.4(\mathrm{C}=\mathrm{O}), 74.0$, $73.6(\mathrm{HCOC}=\mathrm{O}), 66.6\left(\mathrm{H}_{2} \underline{\mathrm{COC}}=\mathrm{O}\right) 38.8\left(\mathrm{HCCH}_{2} \mathrm{O}\right)$ $34.5,34.4\left(\mathrm{O}=\mathrm{CCH}_{2}\right), 25.5\left(\mathrm{O}=\mathrm{CCH}_{2} \underline{\mathrm{CH}_{2}}\right), 31.9,31.8$, $30.5,29.7,29.4,28.9,25.1,23.9,23.0,22.7\left(\mathrm{CH}_{2} \underline{\mathrm{CH}}_{2}\right)$, 14.1, $11.0\left(\mathrm{CH}_{2} \underline{\mathrm{CH}_{3}}\right)$.

\section{RESULTS AND DISCUSSION}

\section{One-pot Synthesis of Polyhydroxy Estolide from Oleic Acid}

Estolide with multiple hydroxyl groups was prepared from oleic acid in accordance with a onepot method that we have reported earlier (Hoong et al., 2017) but with reaction time shortened to 24 hr instead of $72 \mathrm{hr}$. Figure 1 illustrates the one-pot reaction between oleic acid and $\mathrm{H}_{2} \mathrm{O}_{2}$ that yielded PE. Generally, oleic acid was reacted with only $\mathrm{H}_{2} \mathrm{O}_{2}$ at $80^{\circ} \mathrm{C}$ for about $24 \mathrm{hr}$ and Figure 2 illustrates the experimental setup for the reaction. Oleic acid was converted to peroxy acid by $\mathrm{H}_{2} \mathrm{O}_{2}$, which subsequently converted oleic acid to epoxidised oleic acid. Thereafter, the epoxidised oleic acid was ring-opened by another oleic acid, which yielded estolide with hydroxyl groups. OOC analysis conducted on PE showed a value of $0.05 \%$, which indicated that almost all the epoxide groups generated during the reaction were transformed to other functionalities.

Additionally, IV analysis performed on PE revealed that the product had IV of $3 \mathrm{~g} \mathrm{I}_{2} 100 \mathrm{~g}^{-1}$ sample, which indicated that almost all the alkene 
groups of oleic acid were transformed to either ester or hydroxyl groups. The newly formed hydroxyl groups of PE were detected through OHV analysis that recorded a value of $170 \mathrm{mg} \mathrm{KOH} \mathrm{g}^{-1}$. Moreover, ${ }^{1} \mathrm{H}$ NMR analysis confirmed the formation of hydroxyl and ester groups of PE because similar ${ }^{1} \mathrm{H}$ NMR spectrum of PE was observed in literature (Hoong et al., 2017). Furthermore, GPC analysis (Figure 3) showed that the prepared PE consisted of $72 \%$ estolide and $28 \%$ of 9,10-dihydroxystearic acid, which is the monomer of PE. The average molecular weight of PE was about $700 \mathrm{Da}$.

\section{Synthesis of OAE}

According to literature (Salih et al., 2013), base oil that contain hydroxyl group is more susceptible to oxidation degradation than those without hydroxyl group. Consequently, the hydroxyl groups of the prepared PE were end-capped with organic acids to enhance the oxidation stability of the base oil. Various organic acids were employed to end-capped the hydroxyl groups of PE that included linear and branched organic acids as shown in Figure 4.

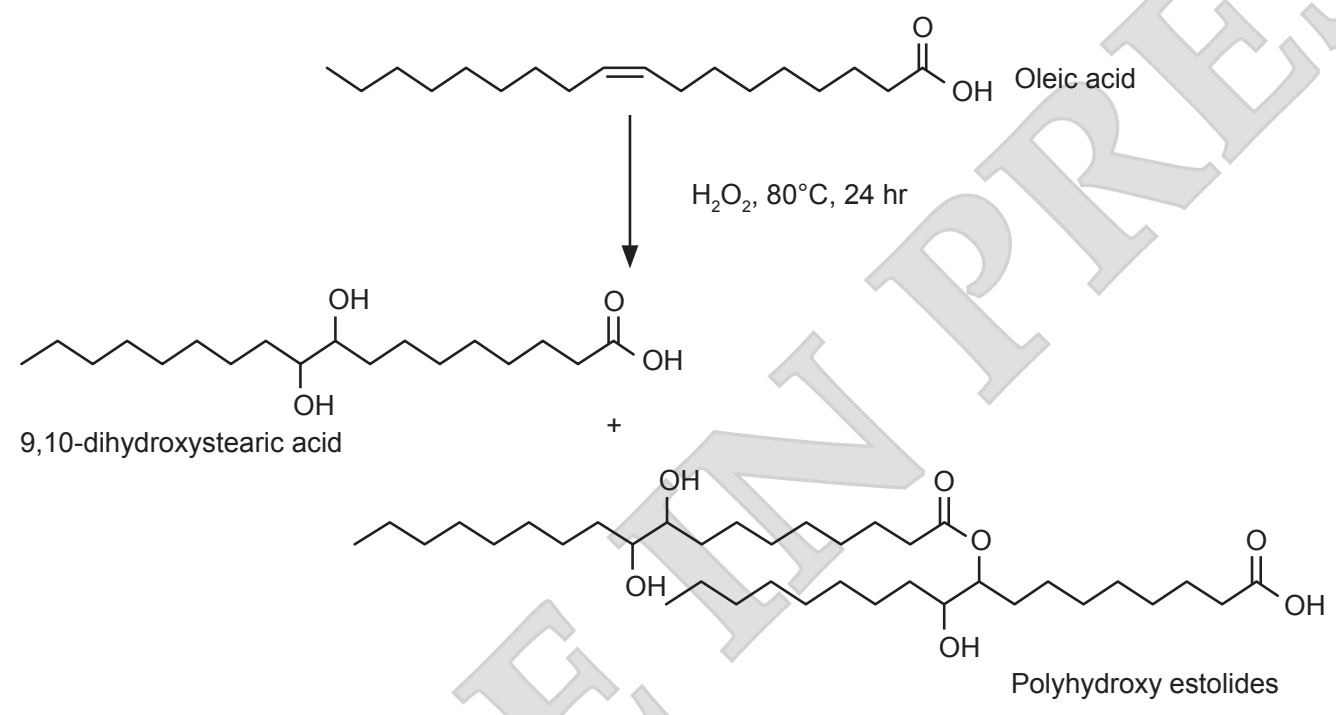

Figure 1. One-pot reaction between oleic acid and hydrogen peroxide $\left(\mathrm{H}_{2} \mathrm{O}_{2}\right)$ that yielded polyhydroxy estolide.

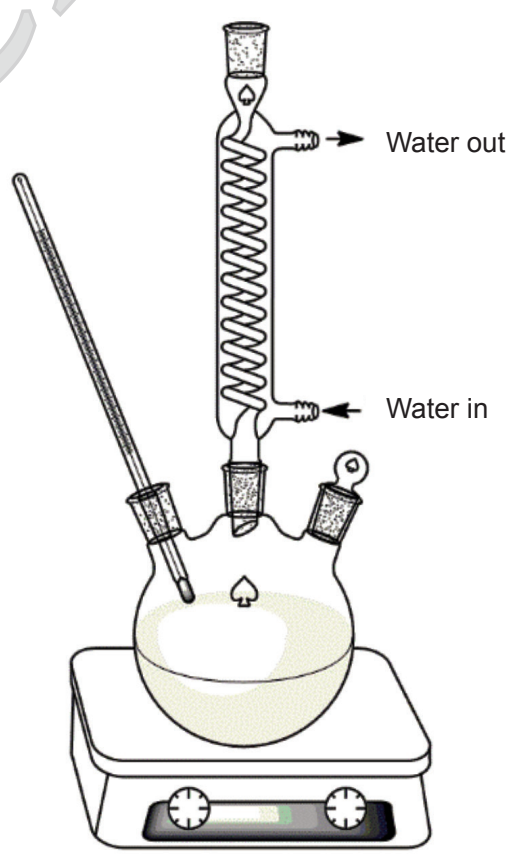

Figure 2. Experimental setup for synthesis of polyhydroxy estolide from oleic acid. 


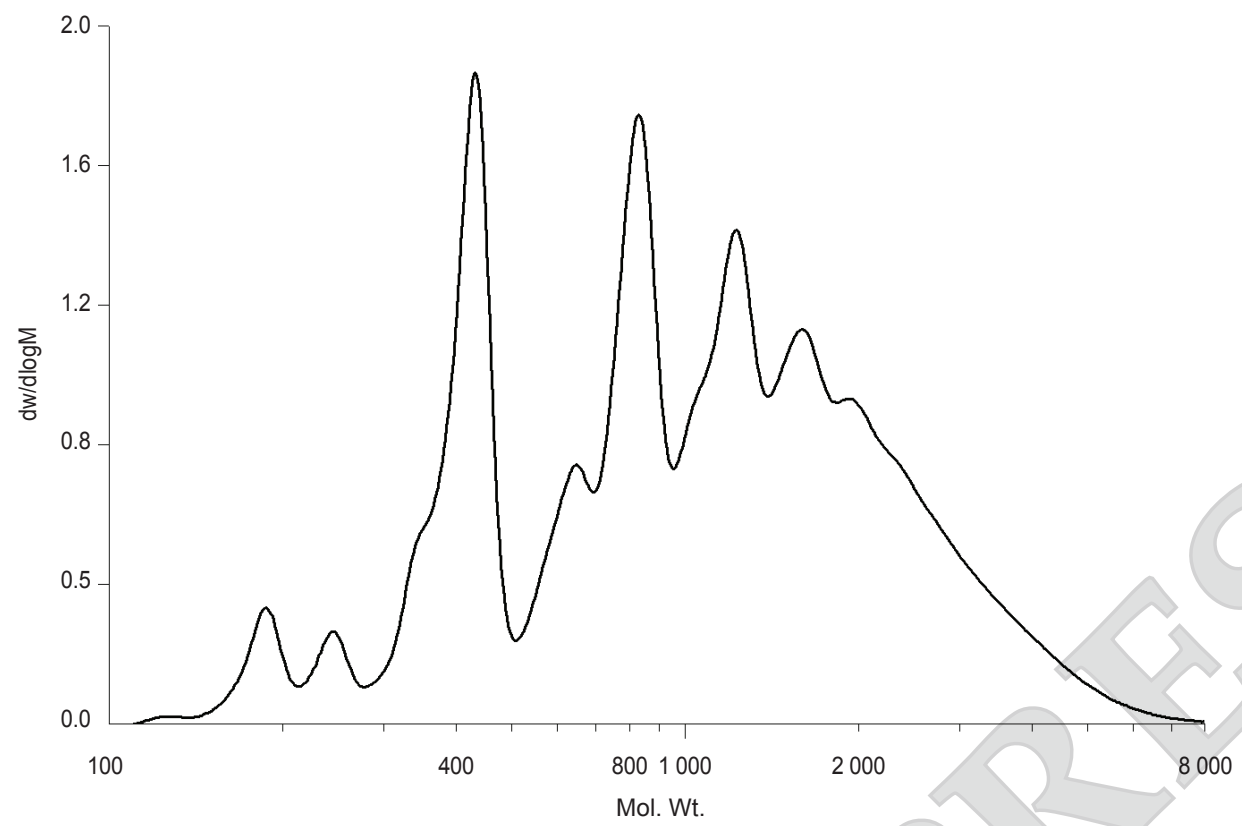

Figure 3. The gel permeation chromatography (GPC) of polyhydroxy estolide.
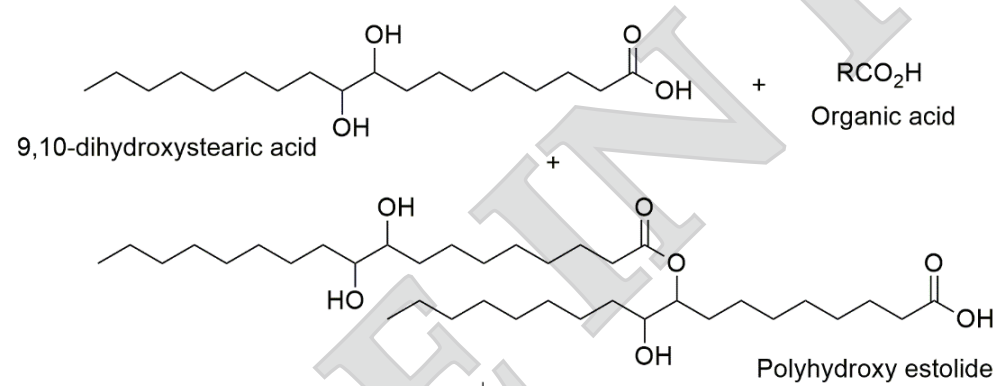

Esterification with organic acid

Z31086, \& hiburs

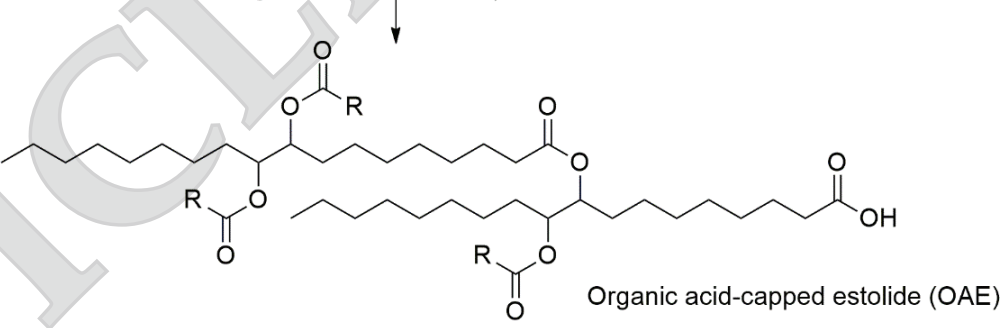

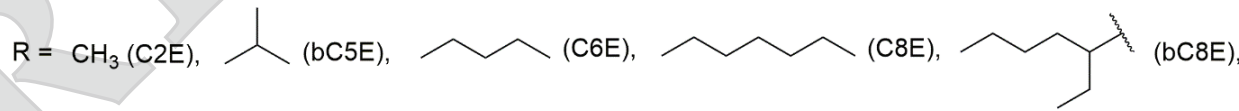

(C12E),

(C18E)

Figure 4. Esterification of polyhydroxy estolide with various organic acids.

The hydrocarbon chain length of linear organic acid used to end-capped PE was varied from short to long chain length. This exercise was meant to evaluate the effect of hydrocarbon chain length of organic acid on the physicochemical properties of resultant lubricant base oil. Selected linear organic acids were acetic acid (C2), C6, C8, C12 and C18.
Literature reported that base oils made of branched molecule showed lower pour point in comparison with linear molecule (Liu et al., 2019). Therefore, branched organic acids were included in this study to evaluate the effect of branched moiety on the properties of base oil. Branched organic acids selected for this study are bC5 and bC8. 
The esterification between PE and organic acids that generate $\mathrm{OAE}$ was conducted without catalyst at temperature between $150^{\circ} \mathrm{C}-210^{\circ} \mathrm{C}$, depending on the boiling point of the organic acid and Figure 5 illustrates the experimental setup for the reaction. For esterification with acetic anhydride, the reaction was carried out at $150^{\circ} \mathrm{C}$ with xylene as the solvent to remove water from the reaction system. While for esterification with the rest of organic acids, reaction temperature was set at $210^{\circ} \mathrm{C}$ to accelerate the reaction. For most of the reaction, an excess amount of organic acid in regard to hydroxyl groups of PE was used in the reaction, in order to end-cap all the hydroxyl groups to form ester groups. The targeted $\mathrm{OHV}$ of the OAE should be less than $10 \mathrm{mg} \mathrm{KOH} \mathrm{g}^{-1}$ and all the prepared OAE achieved OHV less than the targeted value, which indicated that almost all the hydroxyl groups were end-capped with organic acid. For reaction conducted with lauric and stearic acids, the amount of organic acid employed was $50 \% \mathrm{w} / \mathrm{w}$ of PE because excess lauric and stearic acids were difficult to be removed from the product through vacuum distillation.

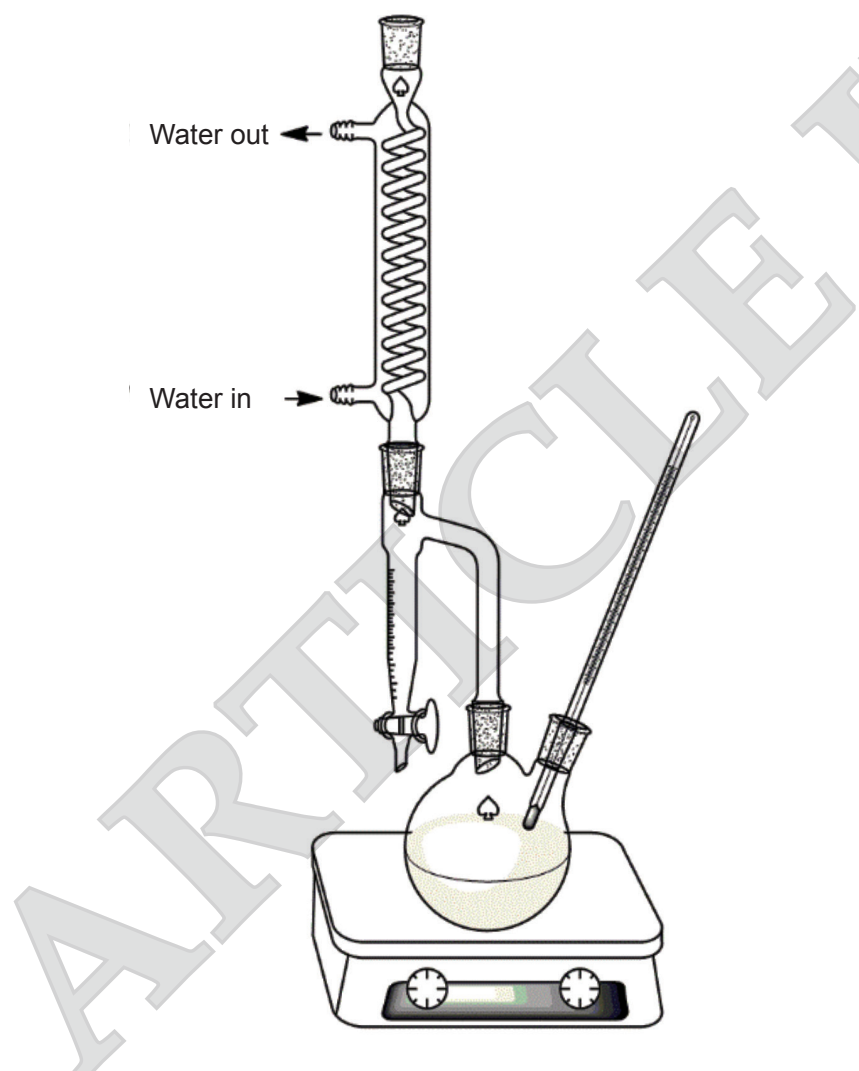

Figure 5. Experimental setup for esterification of between PE and organic acids.

${ }^{1} \mathrm{H}$ NMR analysis of product supported the results of OHV analysis that almost all the hydroxyl groups of PE were end-capped by organic acid to form ester groups. For example, ${ }^{1} \mathrm{H}$ NMR spectrum of hexanoic end-capped estolide (Figure 6a) showed a new peak at $4.97 \mathrm{ppm}$, which correlated to a methine proton attached to an ester group that was formed when hexanoic acid end-capped the hydroxyl group of PE. Additionally, two peaks at 3.57-3.33 ppm in the ${ }^{1} \mathrm{H}$ NMR spectrum of PE (Figure $6 b)$ that correlated to methine protons attached to hydroxyl groups of PE were not observed in the ${ }^{1} \mathrm{H}$ NMR spectra of OAE, which clearly suggested that almost all the hydroxyl groups were converted to ester groups.

\section{Synthesis of 2-ethylhexyl Ester of Organic Acid- capped Estolides}

The synthesised OAE have carboxylic acid groups that are weak acid and may cause corrosion problem to metallic surfaces they intend to protect especially at high operating temperature (Owuna et al., 2020). Therefore, carboxylic group of OAE need to be transformed to ester group in order to impart better qualities to the lubricant base oil. The conversion of carboxylic acid group of OAE to ester group was conducted through esterification with an alcohol. The alcohol selected for the esterification reaction was $2 \mathrm{EH}$ because literature reported (Moser et al., 2007) that branched alcohols such as $2 \mathrm{EH}$ imparted lower pour points to lubricant made with them as compared to linear alcohols.

The esterification between $\mathrm{OAE}$ and $2 \mathrm{EH}$ was carried out without catalyst at $210^{\circ} \mathrm{C}$ for about $8 \mathrm{hr}$ as shown in Figure 7. The experimental setup as shown in Figure 5 was used for the esterification reaction. The $2 \mathrm{EH}$ was used in excess relatively to carboxylic acid group of OAE with the aim of converting all the carboxylic acid groups to esters and to push the reaction forward. The reaction was followed by monitoring the AV of reaction mixture and the targeted $\mathrm{AV}$ of product should be less than $2 \mathrm{mg} \mathrm{KOH} \mathrm{g}^{-1}$ to ensure that the resultant base oil has good lubricant properties. AV analysis performed on all the prepared 2-ethylhexyl ester of organic acidcapped estolides (2EH-OAE) showed AV less than $2 \mathrm{mg} \mathrm{KOH} \mathrm{g}^{-1}$, which indicated that the reaction was successful.

The result of AV analysis was supported by FTIR analysis conducted on 2EH-OAE. As an example, the FTIR spectrum of 2-ethylhexyl ester of octanoic acid-capped estolide (2EH-C8E) (Figure 8) showed a narrow band at $1735 \mathrm{~cm}^{-1}$ that indicated the presence of ester group in the product, while no band associated with carboxylic acid group at 1710 $\mathrm{cm}^{-1}$ was observed, which suggested that all the carboxylic acid groups were transformed to ester groups. Additionally, ${ }^{13} \mathrm{C}$ NMR analysis on products also did not detect peak associated with carboxylic acid group at $179 \mathrm{ppm}$ but instead it detected peak at $174 \mathrm{ppm}$, which indicated the presence of ester group. 


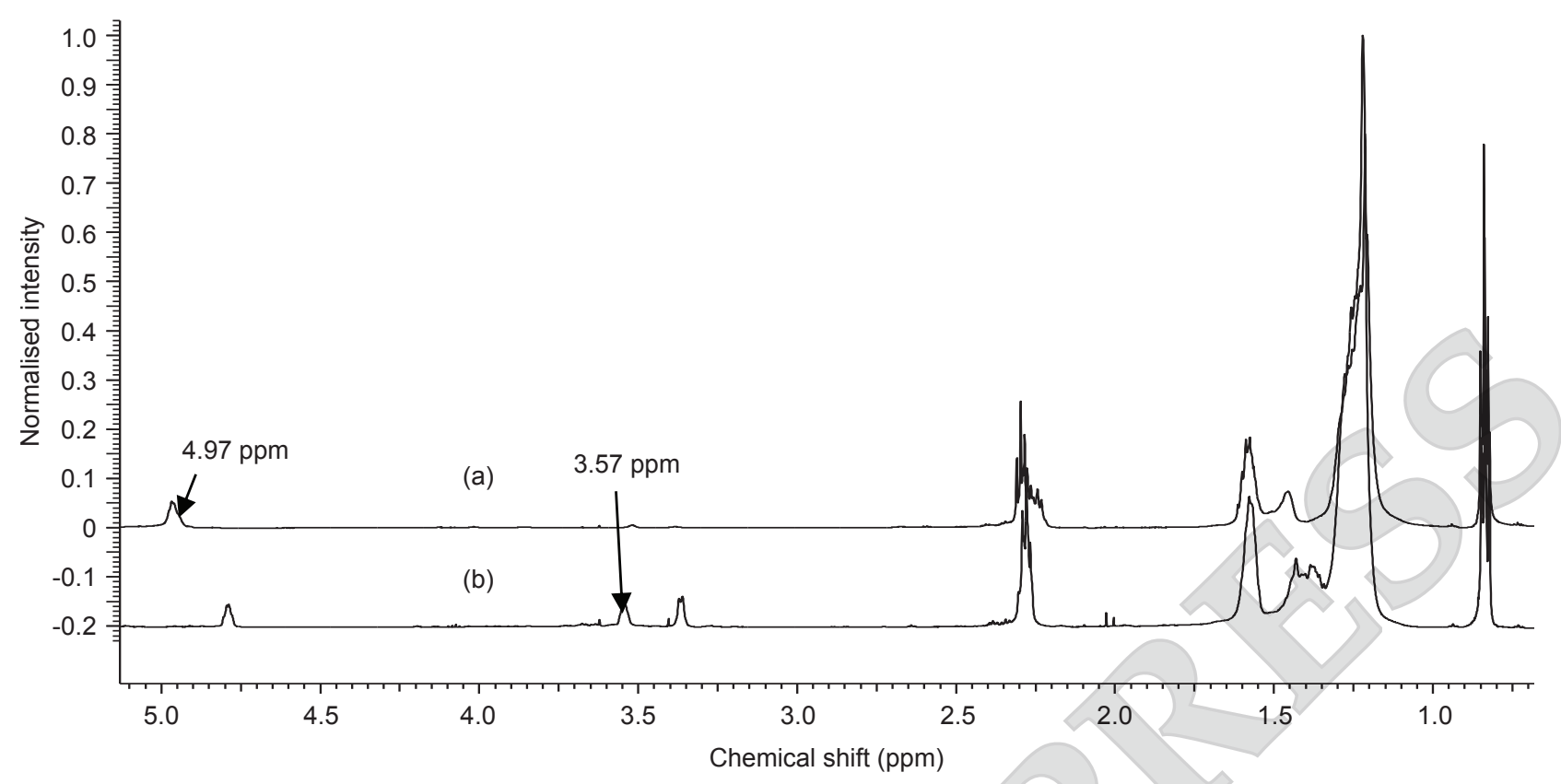

Figure $6 .{ }^{1} H$ NMR spectrum of (a) hexanoic acid-capped estolide, and (b) polyhydroxy estolide (PE).

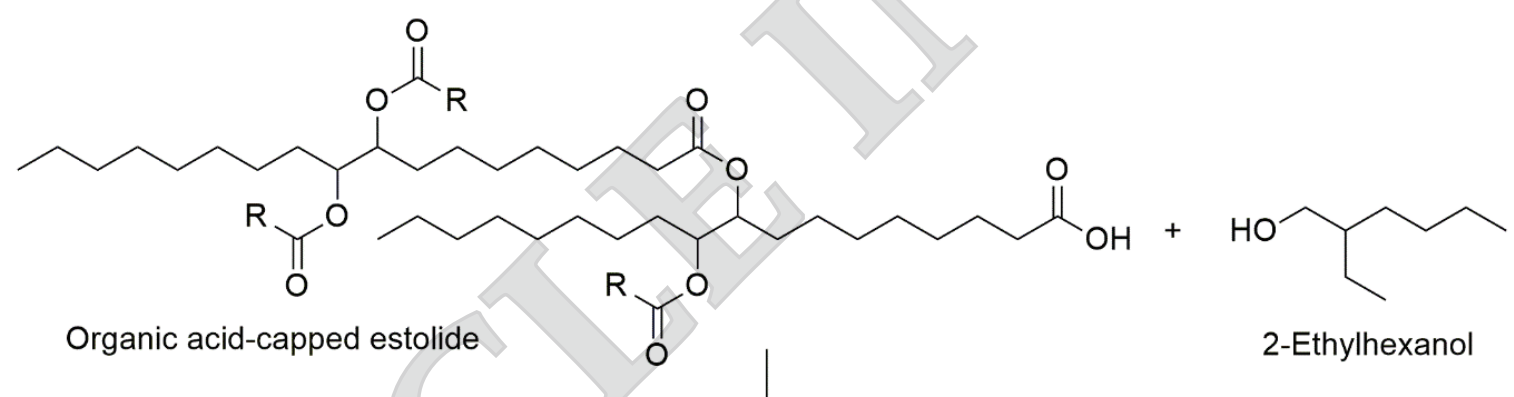

$210^{\circ} \mathrm{C}, 8 \mathrm{hr}$

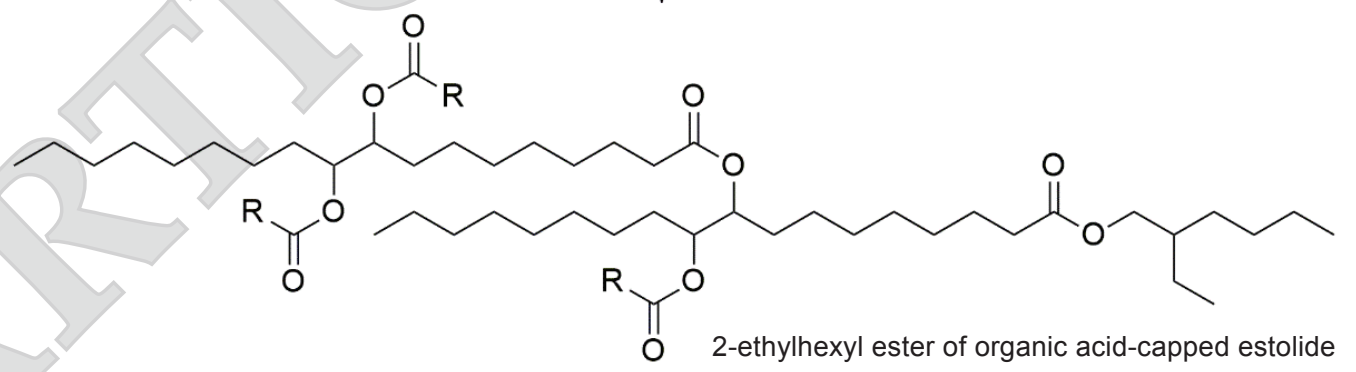

$\mathrm{R}=\mathrm{CH}_{3}(2 \mathrm{EH}-\mathrm{C} 2 \mathrm{E}), \lambda(2 \mathrm{EH}-\mathrm{bC} 5 \mathrm{E})$

$\curvearrowright(2 \mathrm{EH}-\mathrm{C} 6 \mathrm{E}), \curvearrowright(2 \mathrm{EH}-\mathrm{C} 8 \mathrm{E})$,<smiles>CCCCC(CC)C(C)C</smiles>

(2EH-bC8E),

(2EH-C12E),

(2EH-C18E)

Figure 7. Esterification of organic acid-capped estolide with 2-ethylhexanol (2EH). 


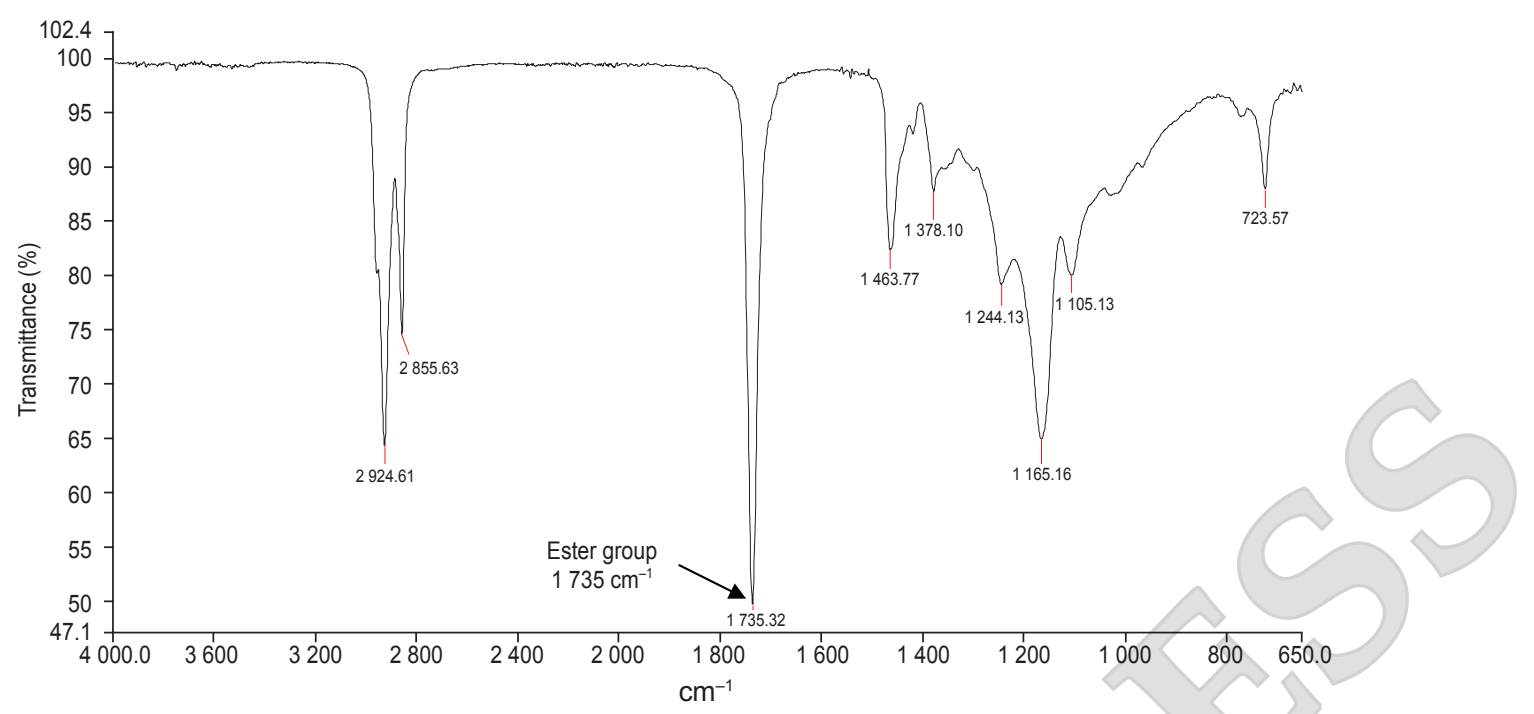

Figure 8. FTIR spectrum of 2-ethylhexyl ester of octanoic end-capped estolide (2EH-C8E).

Evaluation of 2-ethylhexyl Ester of Organic Acidcapped Estolides as Lubricant Base Oil

The synthesised 2EH-OAE were evaluated as lubricant base oil in accordance with typical specifications of commercial lubricant base oil which included kinematic viscosity, pour point, oxidation stability and wear prevention. For the evaluation, a commercial product was included in the study, which served as a benchmark for commercially acceptable lubricant properties. The commercial product was a biolubricant base oil consisted of pentaerythritol tetraoleate, prepared from esterification between pentaerythritol and oleic acid. The results of the physicochemical evaluation of all prepared 2EH-OAEs are shown in Table 1.

One of the main purposes of chemical modification performed on plant oils is to improve the pour point of plant oil-based lubricants to a level comparable to synthetic lubricants that they can be used in arctic condition. As a result of chemical alteration performed on oleic acid, majority of the synthesised 2EH-OAE products showed improved low temperature properties with pour points that were lower than most of commoditised plant oils such as soyabean oil and canola oil. For example, $2 \mathrm{EH}-\mathrm{C} 2 \mathrm{E}$ recorded a pour point of $-21^{\circ} \mathrm{C}$, which was lower than the pour point of canola oil at $-18^{\circ} \mathrm{C}$ (Asadauskas and Erhan, 1999).

Referring to pour points of 2EH-OAE in Table 1, we noticed there was a strong correlation between the chain length of organic acid employed to endcap the hydroxyl group of PE and the pour point of the resultant 2EH-OAE. For example, when organic acid with longer chain length such as $\mathrm{C} 8$ was used to make $2 \mathrm{EH}-\mathrm{C} 8 \mathrm{E}$, the product showed a pour point of $-33^{\circ} \mathrm{C}$, which was lower than pour point of $2 \mathrm{EH}-\mathrm{C} 2 \mathrm{E}\left(-21^{\circ} \mathrm{C}\right)$ that was made with $\mathrm{C} 2$. This observation suggested that the longer chain length of $\mathrm{C} 8$ contributed to the lower pour point. Thus, as anticipated, 2EH-C12E displayed even lower pour point of $-36^{\circ} \mathrm{C}$ as it was made with longer chain organic acid namely $\mathrm{C} 12$ in comparison with C8.

TABLE 1. PHYSICOCHEMICAL PROPERTIES OF 2-ETHYLHEXYL ESTER OF ORGANIC ACIDCAPPED ESTOLIDES

\begin{tabular}{|c|c|c|c|c|c|c|}
\hline \multirow{2}{*}{ Product } & \multicolumn{2}{|c|}{$\mathrm{KV}$} & \multirow{2}{*}{ VI } & \multirow{2}{*}{$\operatorname{PP}\left({ }^{\circ} \mathrm{C}\right)$} & \multirow{2}{*}{ OOT $\left({ }^{\circ} \mathrm{C}\right)$} & \multirow{2}{*}{ WSD (mm) } \\
\hline & $40^{\circ} \mathrm{C}, \mathrm{cSt}$ & $100^{\circ} \mathrm{C}, \mathrm{cSt}$ & & & & \\
\hline 2EH-C2E & 295 & 40 & 189 & -21 & 200 & 0.715 \\
\hline $2 \mathrm{EH}-\mathrm{bC} 5 \mathrm{E}$ & 269 & 35 & 177 & -24 & 196 & 0.757 \\
\hline 2EH-C6E & 285 & 37 & 180 & -24 & 198 & 0.692 \\
\hline 2EH-C8E & 274 & 35 & 175 & -33 & 198 & 0.663 \\
\hline $2 \mathrm{EH}-\mathrm{bC} 8 \mathrm{E}$ & 222 & 28 & 163 & -36 & 193 & 0.734 \\
\hline $2 \mathrm{EH}-\mathrm{C} 12 \mathrm{E}$ & 129 & 20 & 177 & -36 & 197 & 0.631 \\
\hline $2 \mathrm{EH}-\mathrm{C} 18 \mathrm{E}$ & 124 & 19 & 173 & -6 & 196 & 0.612 \\
\hline $\mathrm{C} 1$ & 63 & 12 & 190 & -21 & 150 & 0.648 \\
\hline
\end{tabular}

Note: KV - kinematic viscosity; VI - viscosity index; PP - pour point; OOT - oxidation onset temperature; WSD - four-ball wear scar diameter.

$\mathrm{C} 1$ = commercial biolubricant (pentaerythritol tetraoleate). 
The reason for this outcome was due to the ability of C12 to fully cover the alkyl chain of PE beyond the hydroxyl group that it end-capped and thus, disrupted the intermolecular stacking of estolide molecules, which inhibited crystal formation, and consequently contributed to the low pour point of the product. Conversely, when shorter chain organic acid such as $\mathrm{C} 2$ was used to end-cap the hydroxyl group of $\mathrm{PE}$ as in 2EH-C2E, the short chain length of $\mathrm{C} 2$ was not able to cover the entire alkyl chain length of PE and this allowed alkyl stacking interaction to occur, which eventually resulted in crystal formation and higher pour point value of $2 \mathrm{EH}-\mathrm{C} 2 \mathrm{E}$ at $-21^{\circ} \mathrm{C}$.

This observation is contrarily to general knowledge on ester-based lubricants, which dictates that longer alkyl chain length will impart higher pour point to ester-based lubricant because longer chain length has higher efficiency to pack and form wax-like material than shorter chain length (Stepina et al., 1992). The observed divergence from the norm occurred because the molecular structure of prepared 2EH-OAE is a branched ester unlike mostly linear ester-based lubricant, and the endcapping of hydroxyl group of PE with an organic acid of proper chain length, further disrupted crystal formation that resulted in lower pour point. Similar observation was reported in literature (Isbell, 2011).

Nonetheless, when C18 was used to end-cap hydroxyl groups of $\mathrm{PE}$, the resultant product $2 \mathrm{EH}-$ $\mathrm{C} 18 \mathrm{E}$ exhibited the highest pour point $\left(-6^{\circ} \mathrm{C}\right)$ among prepared products. This was due to the long alkyl chain of C18 that exceeded the length of alkyl chain beyond the hydroxyl group of PE that it endcapped and the extra length of C18 encouraged intermolecular stacking and crystal formation, which led to higher pour point of the product.

The use of branched organic acids had significant effect on the pour point of products made with them, in which products made with branched acids recorded lower pour points than their linear counterpart. This observation was made evidence when comparing pour point of $2 \mathrm{EH}-\mathrm{bC} 8 \mathrm{E}$ with $2 \mathrm{EH}-\mathrm{C} 8 \mathrm{E}$. The branched structure of $\mathrm{bC} 8$ has higher efficacy to obstruct stacking of molecules and avert crystal formation, which resulted in lower pour point than linear C8 (Isbell, 2011). Overall, all the prepared 2EH-OAE exhibited pour points lower or equivalent to commercial product except for $2 \mathrm{EH}-$ $\mathrm{C} 18 \mathrm{E}$, which suggested that most of the 2EH-OAE are good candidates for lubricant base oil.

Another objective of chemical modification conducted on plant oil derivatives is to improve the oxidation stability of plant oil-based lubricants, which is considered inferior in comparison with mineral oil-based lubricants. The oxidation stability of base oil can be evaluated by measuring the OOT of base oil by using pressure differential scanning calorimetry (PDSC) method. Referring to Table 1, the PDSC analysis results showed that the prepared
2EH-OAE had OOT values between $193^{\circ} \mathrm{C}-200^{\circ} \mathrm{C}$, which were remarkably higher than OOT value of the commercial sample. This indicated that the chemical modification performed on the alkene group of oleic acid was able to substantially improve the oxidation stability of resultant base oils, in comparison with commercial samples that contain alkene groups. The observation suggested that conversion of alkene group to ester group can enhance the oxidation stability of base oil made from plant oil derivatives that contain unsaturation sites. Furthermore, the OOT results showed that products made with branched organic acid such as $2 \mathrm{EH}-\mathrm{bC} 8 \mathrm{E}$ registered a lower OOT value $\left(193^{\circ} \mathrm{C}\right)$ than product made with linear organic acid $\left(2 \mathrm{EH}-\mathrm{C} 8 \mathrm{E}, 198^{\circ} \mathrm{C}\right)$, which indicated that products made with linear organic acids were more stable oxidatively.

One of the important attributes of lubricant is its ability to reduce friction and prevent wear on surfaces, which contributes to longer lifespan of machineries. The anti-wear properties of lubricants can be measured by a four-ball method in accordance with ASTM 4172 and usually test result is reported as WSD, in which lubricant with better anti-wear properties would exhibit smaller WSD. As shown in Table 1, product 2EH-C18E exhibited the smallest WSD among all prepared 2EH-OAE, which suggested that it had the best anti-wear properties. The good anti-wear attribute of 2EHC18E was most likely contributed by the long chain linear C18 employed to end-cap the hydroxyl group of PE as similar observation was reported by Salih et al. (2011).

Thus, for products that were made with shorter chain linear organic acids such as 2EH-C12E and 2EH-C8E, the WSD recorded for these products were slightly larger $(0.631-0.663 \mathrm{~mm})$ than $2 \mathrm{EH}$ C18E, which reflected the negative effect of shorter chain organic acids on the anti-wear properties. For product made with even shorter chain fatty acid namely 2EH-C6E, the WSD was even larger $(0.692$ $\mathrm{mm})$. Eventually, the product 2EH-C2E prepared with the shortest chain length organic acid (C2) exhibited the largest WSD among 2EH-OAE made with linear organic acids.

In comparison to products made with linear organic acids, the WSD for products made with branched organic acids were even larger, which suggested that they provided less wear prevention than products made with linear organic acids. This observation was made evidence when comparing WSD of $2 \mathrm{EH}-\mathrm{bC} 8 \mathrm{E}$ with $2 \mathrm{EH}-\mathrm{C} 8 \mathrm{E}$, in which $2 \mathrm{EH}-$ bC8E showed larger WSD due to its branched moiety as reported in literature (Sharma and Erhan, 2013). Nevertheless, three products namely 2EHC8E, 2EH-C12E and 2EH-C18E exhibited anti-wear properties similar or better than the commercial product used as benchmark. 
Viscosity is a crucial physical property of base oil for lubricant. In the context of lubrication, viscosity of lubricant is commonly measured by kinematic viscosity and can be classified according to ISO VG system, which uses the kinematic viscosity of a fluid at $40^{\circ} \mathrm{C}$ as the reference point for classification. Referring to Table 1, the viscosity of 2EH-OAE was inversely proportionate to the chain length of organic acid employed in their syntheses. For example, product $2 \mathrm{EH}-\mathrm{C} 2 \mathrm{E}$, which was made with the shortest chain length organic acid showed the highest kinematic viscosity of $295 \mathrm{cSt}$, while product made with the longest chain length organic acid (2EH-C18E) exhibited the lowest viscosity of 124 cSt. Additionally, product made with branched acid exhibited slightly lower viscosity when compared with its linear counterpart and this was made evidence when comparing product $2 \mathrm{EH}-\mathrm{bC} 8 \mathrm{E}$ to 2EH-C8E. Similar observation on the effect of alkyl chain length on the viscosity of OAE was reported by Isbell (2011).

The prepared 2EH-OAE can be divided into two groups based on their kinematic viscosity at $40^{\circ} \mathrm{C}$. The first group consisted of product $2 \mathrm{EH}-$ C12E and 2EH-C18E, which recorded kinematic viscosity between 124-129 cSt and can be classified as ISO VG 150 base oil. The second group consisted of the rest of the 2EH-OAE that were made with C2 to C8 organic acids, which displayed kinematic viscosity between $200-300 \mathrm{cSt}$ at $40^{\circ} \mathrm{C}$. Product 2EH-bC8E can be classified as ISO VG 220 base oil while the rest of products can be classified as ISO VG 320 base oil. All the prepared products with viscosity between ISO VG 150 and ISO VG 320 can be used as lubricant for gears and bearings, which require base oil with relatively high viscosity (Pirro et al., 2016).

VI is an indication of a lubricant change in viscosity relative to temperature change. It is a classification to describe the viscosity-temperature relationship of lubricant. Lubricant with higher VI indicates that the viscosity of the lubricant is less affected by temperature fluctuations. Generally, any base oil with VI more than 100 is considered to have good viscosity-temperature consistency profile (Tanveer and Prasad, 2006). As shown in Table 1, all the prepared products exhibited VI between163-189 and this result suggested that all the prepared 2EHOAE have excellent VI quality.

\section{CONCLUSION}

The present study has shown that estolides esters can be made from oleic acid through chemical modification performed on alkene group of oleic with $\mathrm{H}_{2} \mathrm{O}_{2}$ that generated PE. Subsequently, the hydroxyl groups of PE were end-capped by various organic acids in order to evaluate the effect of organic acid moiety on the lubricant properties of resultant base oil. Afterward, the carboxylic acid group of OAE was converted to ester group with $2 \mathrm{EH}$ to further enhanced its lubricant properties.

Generally, the chemical modifications conducted on oleic acid that eventually generated estolides esters, not only significantly improved the low temperature properties of estolide esters but also remarkably enhanced the oxidation stability of estolide esters in comparison with the starting material and a commercial product. These results highlighted that the two major drawbacks associated with plant oil-based lubricants namely high pour point and poor oxidation stability can be resolved by converting oleic acid to estolide esters.

In particular, the chain length of organic acid that end-capped the hydroxyl group of PE strongly affected the properties of base oil. Products made with linear lauric acid (2EH-C12E) and branched 2-ethylhexanoic acid (2EH-bC8E), respectively exhibited the best pour point $\left(-36^{\circ} \mathrm{C}\right)$, while products made with shorter or longer chain length organic acids showed higher pour points. Furthermore, products made with longer chain organic acid recorded better anti-wear properties and lower kinematic viscosity than products made with shorter chain length organic acid. Additionally, the oxidation stability of products made with linear organic acid were higher than those made with branched organic acids.

Most of the 2EH-OAE prepared in this study have physicochemical properties that comply with standard specifications of hydraulic oil, gear oil and stern tube lubricants, which indicated that most of the prepared 2EH-OAE are suitable to be used as base oil for lubricants.

\section{ACKNOWLEDGEMENT}

We would like to thank the Director-General of MPOB for permission to publish this article. We are grateful to Ahmad Lutfi Md Yusof, Zuraidah Kamarudin, Sapiah Hashim, Selasiah Abdullah, Mohd Taib Samsudin, Norizan Ali and Makmor Abd. Wahab for all the analyses conducted. We would like to express our gratitude to IOI Acidchem $\mathrm{Sdn}$. Bhd. for providing free sample of oleic acid (PALMAC 750).

\section{REFERENCES}

Abi-Akar, H (2017). Performance and technical requirements of low-environment impact lubricants. Environmentally Friendly and Biobased Lubricants (Sharma, B K and Biresaw, G eds.). CRC Press, Boca Raton, USA. p. 102-103. 
Adhvaryu, A; Liu, Z and Erhan, S Z (2005). Synthesis of novel alkoxylated triacylglycerols and their lubricant base oil properties. Ind. Crops Prod., 21: 113119.

Armylisas, A H N; Hazirah, M F S; Yeong, S K and Hazimah, A H (2017). Modification of olefinic double bonds of unsaturated fatty acids and other vegetable oil derivatives via epoxidation: A review. Grasas $Y$ Aceites, 68: e174.

Asadauskas, S and Erhan, S Z (1999). Depression of pour points of vegetable oils by blending with diluents used for biodegradable lubricants. J. Amer. Oil Chem. Soc., 76: 313-316.

Battersby, N S; Pack, S E and Watkinson, R J (1992). A correlation between the biodegradability of oil products in the CEC L-33-T-82 and modified Sturm tests. Chemosphere, 24: 1989-2000.

Borugadda, V B and Dalai, AK (2018). In-situ synthesis and characterization of biodegradable estolides via epoxidation from canola biodiesel. Lubricants, 6: 94 .

Cecilia, J A; Plata, D B; Saboya, R M A; Luna, F M T; Cavalcante, C L and Castellon, E R (2020). An overview of the biolubricant production process: Challenges and future perspectives. Processes, 8: 257.

Cermak, S C and Isbell, T A (2001). Synthesis of estolides from oleic and saturated fatty acids. J. Amer. Oil Chem. Soc., 78: 557-565.

Cermak, S C and Isbell, T A (2003a). Synthesis and physical properties of estolide-based functional fluids. Ind. Crops Prod., 18: 183-196.

Cermak, S C and Isbell, T A (2003b). Improved oxidative stability of estolide esters. Ind. Crops Prod., 18: 223-230.

Cermak, S C; Brandon, K B and Isbell, T A (2006). Synthesis and physical properties of estolides from lesquerella and castor fatty acid esters. Ind. Crops Prod., 23: 54-64.

Cermak, S C; Bredsguard, J W; Roth, K L; Thompson, T; Feken, K A; Isbell, T and Murray, R E (2015). Synthesis and physical properties of new coco-oleic estolide branched esters. Ind. Crops Prod., 74: 171-177.

Chan, C H; Tang, S W; Mohd, N K; Lim, W H and Yeong, S K (2018). Tribological behavior of biolubricant base stocks and additives. Renew. Sust. Energ. Rev., 93: 145-157.

Chen, J M; Beaufort, M L; Gyurik, L; Dorresteijn, J; Otte, M and Gebbink, R J M K (2019). Highly efficient epoxidation of vegetable oils catalyzed by a manganese complex with hydrogen peroxide and acetic acid. Green Chem., 21: 2436-2447.

Chen, Y Z; Biresaw, G; Cermak, S C; Isbell, T A; Ngo, H L; Chen, L and Durham, A L (2020). Fatty acid estolides: A review. J. Amer. Oil Chem. Soc., 97: 231241.

Erhan, S Z; Sharma, B K and Perez, J M (2006). Oxidation and low temperature stability of vegetable oil-based lubricants. Ind. Crops Prod., 24: 292-299.

Everett, K and Graf, F A J (1971). Handling perchloric acid and perchlorates. CRC Handbook of Laboratory Safety (Steere, N V ed.). CRC Press, Boca Raton, USA. p. 265.

Greco-Duarte, J; Collaco, A C A; Costa, A M M; Silva, L O; Da Silva, JA C; Torres, A G; Fernandez-Lafuente, $\mathrm{R}$ and Freire, D M G (2019). Understanding the degree of estolide enzymatic polymerization and the effects on its lubricant properties. Fuel, 245: 286-293.

Heikal, E K; Elmelawy, M S; Khalil, S A and Elbasuny, N M (2017). Manufacturing of environment friendly biolubricants from vegetable oils. Egypt. J. Pet., 26: 53-59.

Hoong, S S; Norhisham, M S; Maznee, T N T I; Kosheela, D P P; Nurul, A H; Hazimah, A B; Ooi, T L and Salmiah, A (2008). Preparation of a low viscosity and light coloured fatty acid-based polyol. J. Oil Palm Res. (Special Issue-October 2008) 20: 44-52.

Hoong, S S; Yeong, S K and Hazimah, A B (2017). Non-catalytic one-pot synthesis of polyhydroxy estolides from oleic acid. J. Oil Palm Res., 29: 88-96.

Isbell, T (2011). Chemistry and physical properties of estolides. Grasas Y Aceites, 62: 8-20.

Kassfeldt, E and Goran, D (1997). Environmentally adapted hydraulic oils. Wear, 207: 41-45.

Kim, H J; Choi, N K; Kim, Y H; Kim, H R; Lee, J S and Kim, I H (2019). Immobilized lipase-catalyzed esterification for synthesis of trimethylolpropane triester as a biolubricant. Renew. Energy, 130: 489-494.

Liu, S B; Saha, B and Vlachos, D G (2019). Catalytic production of renewable lubricant base oils from bio-based 2-alkylfurans and enals. Green Chem., 21: 3606-3614.

Luther, R (2007). Lubricants in the environment. Lubricants and Lubrication (Mang, $\mathrm{T}$ and Dresel, W eds.). Wiley-VCH, Weinheim, Germany. p. 120-123. 
Lv, N N; He, W; Fang, Z; Sun, Q; Qiu, C H and Guo, K (2017). Epoxidation of methyl oleate and subsequent ring-opening catalyzed by lipase from Candida sp. 99-125. Eur. J. Lipid Sci. Technol., 120: 1700257.

Moser, B R; Sharma, B K; Doll, K M and Erhan, S Z (2007). Diesters from oleic acid: Synthesis, low temperature properties and oxidation stability. J. Amer. Oil Chem. Soc., 84: 675-680.

Mubofu, E B (2016). Castor oil as a potential renewable resource for the production of functional materials. Sustain. Chem. Process., 4: 11.

Nowak, P; Kucharska, K and Kaminski, M (2019). Ecological and health effects of lubricant oils emitted into the environment. Int. J. Environ. Res. Public Health, 16: 3002.

Nowicki, J; Drabik, J; Woszczynski, P; Gebura, K; Nowakowska-Bogdan, E and Kozdrach, R (2019). Tribological characterization of plant oil derived fatty acid esters of higher polyols: Comparative experimental study. Lubr. Sci., 31: 61-72.

Owuna, F J; Dabai, M U; Sokoto, M A; Dangoggo, S M; Bagudo, B U; Birnin-Yauri, UA;Hassan, LG;Sada, I; Abubakar, A L and Jibrin, M S (2020). Chemical modification of vegetable oils for the production of biolubricant using trimethylolpropane: A review. Egypt. J. Pet., 29: 75-82.

Parveez, G K A; Hishamuddin, E; Loh, S K; Ong-Abdullah, M; Salleh, K M; Bidin, M N I Z; Sundram, S; Hasan, Z A A and Idris, Z (2020). Oil palm economic performance in Malaysia and R\&D progress in 2019. J. Oil Palm Res., 32: 159-190.

Pirro, D M; Webster, M and Daschner, E (2016). Lubrication Fundamentals. CRC Press, Boca Raton, USA. p. 197.

Reeves, C J; Siddaiah, A and Menezes, P L (2017). A review on the science and technology of natural and synthetic biolubricants. J. Bio Tribo Corros., 3: 11.

Sammaiah, A; Padmaja, K V and Prasad, R B N (2016). Synthesis and physical properties of novel estolides from dicarboxylic acids and methyl ricinoleate. Eur. J. Lipid Sci. Technol., 118: 486-494.

Salih, N; Salimon, J and Yousif, E (2011). The physicochemical and tribological properties of oleic acid based triesters biolubricants. Ind. Crops Prod., 34: 1089-1096.

Salih, N; Salimon, J; Yousif, E and Abdullah, B M (2013). Biolubricant basestocks from chemically modified plant oils: Ricinoleic acid based-tetraesters. Chem. Cent. J., 7: 128.

Salimon, J; Salih, N and Yousif, E(2010). Biolubricants: Raw materials, chemical modifications and environmental benefits. Eur. J. Lipid Sci. Technol., 112: 519-530.

Salimon, J; Salih, N and Yousif, E (2011). Synthesis, characterization and physicochemical properties of oleic acid ether derivatives as biolubricant basestocks. J. Oleo Sci., 60: 613-618.

Santos, J C; Santos, IM; Conceico, M M; Porto, S L; Trindade, M F; Souza, A G; Prasad, S; Fernandes, J and Araujo, A S (2004). Thermoanalytical, kinetic and rheological parameters of commercial edible plant oils. J. Therm. Anal. Calorim., 75: 419-428.

Sharma, B K and Erhan, S Z (2013). Modified vegetable oil for environmentally friendly lubricant applications. Synthetics, Mineral Oils and Bio-Based Lubricants (Rudnick, L R ed.). CRC Press, Boca Raton, USA. p. 400.

Soni, S and Agarwal, M (2014). Lubricants from renewable energy sources - A review. Green Chem. Lett. Rev., 7: 359-382.

Stepina, V and Vesely, V (1992). Lubricants and Special Fluids. Elsevier, Amsterdam, Netherlands. p. 167.

Tanveer, S and Prasad, R (2006). Enhancement of viscosity index of mineral base oils. Indian J. Chem. Technol., 13: 398-403.

Theissen, H (2010). The German market introduction program for biobased lubricants. Tribology Online, 5: 225-229. 